\title{
Nonequilibrium states of a plasmonic Dicke model with coherent and dissipative surface-plasmon-quantum-emitter interactions
}

\author{
Andrei Piryatinski $\odot,{ }^{1, *}$ Oleksiy Roslyak, ${ }^{2}$ Hao Li $\odot,{ }^{3}$ and Eric R. Bittner $\odot^{3, \dagger}$ \\ ${ }^{1}$ Theoretical Division, Los Alamos National Laboratory, Los Alamos, New Mexico 87545, USA \\ ${ }^{2}$ Department of Physics and Engineering Physics, Fordham University, Bronx, New York 10458, USA \\ ${ }^{3}$ Department of Chemistry, University of Houston, Houston, Texas 77204, USA
}

(Received 4 September 2019; revised manuscript received 27 November 2019; accepted 8 January 2020; published 10 February 2020)

\begin{abstract}
Hybrid photonic-plasmonic nanostructures allow one to engineer coupling of quantum emitters and cavity modes accounting for the direct coherent and environment-mediated dissipative pathways. Using the generalized plasmonic Dicke model, we explore the nonequilibrium phase diagram with respect to these interactions. The analysis shows that their interplay results in the extension of the superradiant and regular lasing states to the dissipative coupling regime and an emergent lasing phase without population inversion having a boundary with the superradiant and normal states. Calculated photon emission spectra are demonstrated to carry distinct signatures of these phases.
\end{abstract}

DOI: 10.1103/PhysRevResearch.2.013141

\section{INTRODUCTION}

In quantum plasmonics, highly polarizable metal nanostructures supporting surface-plasmon modes provide a source of strong enhancement in the photon local density of states, an effect similar to a low- $Q$ optical cavity [1]. Technological flexibility in the design of plasmonic cavities allows one to engineer surface-plasmon states and their interactions with quantum emitters (QEs), e.g., fluorescent dyes or semiconductor nanostructures, leading to potentially desirable cooperative properties [1-3]. The strong (ultrastrong) coupling regimes, when the surface-plasmon- $\mathrm{QE}$ interaction strength exceeds the total cavity losses (becomes comparable to the $\mathrm{QE}$ energy), open new opportunities for nonequilibrium exciton-plasmon-polariton condensation, nonlinear emission, and lasing $[4,5]$.

In many cases, the experimental demonstration of the effect was preceded by theoretical analysis. For instance, theoretical studies of surface-plasmon-induced superradiant and subradiant Dicke states reveal how their frequency and time-domain emission features depend on the cavity geometry, composition, and environment fluctuations [6,7]. Rapid progress in the development of a nanoscale surface-plasmon laser, often referred to as the spaser, has been reported [8-12]. Theoretical analysis of critical phenomena such as Bose-Einstein condensation (BEC) of the surface-plasmon-exciton polaritons in

\footnotetext{
*apiryat@lanl.gov

†ebittner@central.uh.edu
}

Published by the American Physical Society under the terms of the Creative Commons Attribution 4.0 International license. Further distribution of this work must maintain attribution to the author(s) and the published article's title, journal citation, and DOI. plasmonic lattices and arrays $[13,14]$ was followed by reports claiming experimental observation of a thermalized room temperature and nonequilibrium BEC along with polariton lasing $[4,15]$.

In this paper we explore the possibility of engineering quantum critical properties of plasmonic cavities by examining the nonequilibrium phase diagram and associated photon emission spectra with respect to the nature and strength of the surface-plasmon-QE interactions. To do so, we generalize a driven-dissipative Dicke model describing an ensemble of identical two-level QEs coupled to a single bosonic surfaceplasmon mode referred to below as the surface-plasmon cavity mode (SPCM) [Fig. 1(a)]. We incorporate into the model two distinct SPCM-QE interaction pathways which we illustrate in Fig. 1(b): a coherent coupling $\lambda$, which stems from a direct, e.g., dipole-dipole, interaction between the SPCM and a QE, and a dissipative coupling $\eta$, which is facilitated by coupling to a reservoir, which may be either a photon continuum or a dark surface-plasmon mode [illustrated in Fig. 1(a)] interacting in the near field with both the SPCM and QEs. The energy is injected into the cavity incoherently, e.g., via electrical pumping or optical excitation of the QE high excited states followed by phonon-assisted relaxation to the "active" excited state. The SPCM is coupled to the photon continuum facilitating the cavity emission.

The dissipative coupling via photon reservoir was introduced by Lehmberg as the off-diagonal radiative decay terms in the Lindblad operator to describe the superradiant emission from an ensemble of two-level atoms [16]. Subsequently, this approach has been widely used to study the superradiant emission in a large variety of systems $[6,17,18]$. Coupling via a reservoir has been introduced in cascaded quantum systems $[19,20]$ and shown to be important for quantum information applications [21]. The dissipative coupling is also shown to facilitate quantum entanglement [22-24] and can be 


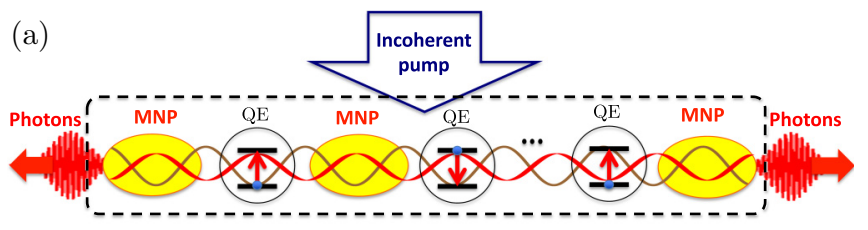

(b)

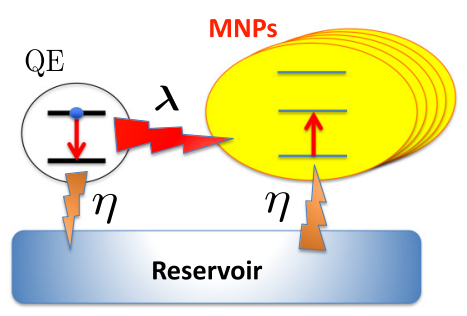

FIG. 1. (a) Example of plasmonic cavity filled with QEs coupled to the SPCM (red wavy line) and dark plasmon mode (brown wavy line), both due to an array of metal nanoparticles (MNPs). The SPCM also interacts with the dark plasmon mode. The QEs are subject to an incoherent pumping and the SPCM is the source of photons emitted outside the cavity. (b) Partitioning of the interaction rate between a $\mathrm{QE}$ and the quantized SPCM states into the coherent $\lambda$ and dissipative $\eta$ quantum exchange rates.

employed to steer open quantum system into nontrivial states $[25,26]$.

The Dicke model provides a fundamental model of cavity QED and has been studied in a context of superradiant phase transition in both equilibrium and nonequilibrium regimes [27]. Its generalized version with imbalanced rotating and counterrotating terms has revealed a rich phase diagram allowing for the superradiant and various lasing states [27-29]. In this paper we demonstrate that the interplay of the coherent and dissipative interactions incorporated into the plasmonic Dicke model results in the extension of the superradiant and regular lasing phases to the dissipative coupling region of the phase diagram and shows an emergent lasing state without population inversion (referred to below as lasing without inversion) having phase boundaries with the superradiant and normal steady states. We demonstrate that the associated photon emission spectra carry distinct spectroscopic signatures of the various phases.

The paper organized as follows. Generalization of the plasmonic Dicke model accounting for the dissipative coupling is presented in Sec. II. In Sec. III the mean field and secondmoment equations of motion are employed to calculate the nonequilibrium phase diagram and to identify the steady states. Associated photon emission spectra are analyzed in Sec. IV. Conclusions are given in Sec. V.

\section{GENERALIZED DICKE MODEL}

The SPCM cavity mode (Fig. 1) is described by Bose operators $\left\{\psi^{\dagger}, \psi\right\}$. Each two-level QE occupying site $n=$ $\overline{1, \mathcal{N}_{0}}$ is characterized by a set of spin operators $\left\{\hat{s}_{n}^{ \pm}=\hat{s}_{n}^{x} \pm\right.$ $\left.i \hat{s}_{n}^{y}, s_{n}^{z}\right\}$, related to the Pauli $\mathrm{SU}(2)$ operators as $\hat{s}_{n}^{j}=\frac{1}{2} \hat{\sigma}_{n}^{j}$, with $j=x, y, z$. Assuming that the QEs and the SPCM have the same resonance energy $\omega_{0}$ and the same coherent quantum exchange rate $\lambda$, we describe the system by the Dicke
Hamiltonian given in units of $\hbar$,

$$
\begin{aligned}
\hat{H}_{D}= & \omega_{0} \hat{\psi}^{\dagger} \hat{\psi}+\omega_{0}\left(\sum_{n=1}^{\mathcal{N}_{0}} \hat{s}_{n}^{z}+\frac{\mathcal{N}_{0}}{2}\right) \\
& +\lambda\left(\hat{\psi}+\hat{\psi}^{\dagger}\right) \sum_{n=1}^{\mathcal{N}_{0}}\left(\hat{s}_{n}^{-}+\hat{s}_{n}^{+}\right) .
\end{aligned}
$$

By taking into account that the plasmonic cavity (Fig. 1) is an open quantum system, we introduce a density operator $\hat{\rho}$ projected on the SPCM and QE space whose time evolution is described by a Liouville equation

$$
\begin{aligned}
\partial_{t} \hat{\rho}= & -i\left[\hat{H}_{D}, \hat{\rho}\right]+\Gamma_{s p} \hat{\mathcal{D}}_{\hat{\psi}}[\hat{\rho}]+\frac{\gamma_{\uparrow}}{2} \sum_{n=1}^{\mathcal{N}_{0}} \hat{\mathcal{D}}_{\hat{s}_{n}^{+}}[\hat{\rho}] \\
& +\frac{\gamma_{\downarrow}}{2} \sum_{n=1}^{\mathcal{N}_{0}} \hat{\mathcal{D}}_{\hat{s}_{n}^{-}}[\hat{\rho}]+\gamma_{\phi} \sum_{n=1}^{\mathcal{N}_{0}} \hat{\mathcal{D}}_{\hat{S}_{n}^{z}}[\hat{\rho}]+\eta \sum_{n=1}^{\mathcal{N}_{0}} \hat{\mathcal{D}}_{\hat{\psi}, \hat{s}_{n}^{+}}[\hat{\rho}] .
\end{aligned}
$$

Here the Lindblad superoperator $\hat{\mathcal{D}}_{\hat{\mathcal{O}}}[\hat{\rho}]=\left(2 \hat{\mathcal{O}} \hat{\rho} \hat{\mathcal{O}}^{\dagger}-\right.$ $\left.\hat{\mathcal{O}}^{\dagger} \hat{\mathcal{O}} \hat{\rho}-\hat{\rho} \hat{\mathcal{O}}^{\dagger} \hat{\mathcal{O}}\right)$, with $\hat{\mathcal{O}}=\left\{\hat{\psi}, \hat{s}_{n}^{-}, s_{n}^{+}, \hat{s}_{n}^{z}\right\}$, acts either within the SPCM or QE subspaces. It describes the SPCM population decay with the rate $2 \Gamma_{s p}$, QE population decay with the rate $\gamma_{\downarrow}$, population gain due to an incoherent pump with the rate $\gamma_{\uparrow}$, and pure dephasing with the rate $\gamma_{\phi}$. All introduced population decay rates include both the radiative and nonradiative contributions. The last term in Eq. (2) partitions the dissipative SPCM and QE interaction with the rate $\eta$ using the Lindblad operator ${ }^{1}$

$$
\begin{aligned}
\hat{\mathcal{D}}_{\hat{\psi}, \hat{s}_{n}^{+}}[\hat{\rho}]= & 2 \hat{\psi} \hat{\rho} \hat{s}_{n}^{+}-\hat{s}_{n}^{+} \hat{\psi} \hat{\rho}-\hat{\rho} \hat{s}_{n}^{+} \hat{\psi} \\
& +2 \hat{s}_{n}^{-} \hat{\rho} \hat{\psi}^{\dagger}-\hat{\psi}^{\dagger} \hat{s}_{n}^{-} \hat{\rho}-\hat{\rho} \hat{\psi}^{\dagger} \hat{s}_{n}^{-} .
\end{aligned}
$$

Equations (1)-(3) constitute our generalization of a drivendissipative plasmonic Dicke model.

Microscopic derivation of this model with the SPCM-QE interactions facilitated by the photon continuum is provided in Appendix A. Associated expressions for the coherent and incoherent coupling rates are given by Eqs. (A42) and (A43) complemented by Eqs. (A27) and (A28), respectively. These equations naturally account for the photon retardation effects allowing one to treat plasmonic cavities with linear size of the order of an optical wavelength, the regime when conventional near-field dipole-dipole approximation breaks down. In the near-field limit, the dissipative interaction (A28) becomes weak, i.e., scales with the distance $r$ between the dipole as $r^{-1}$. Therefore, dropping the dissipative term in the near-field limit, we recover a plasmonic cavity model used in Ref. [14] to study equilibrium exciton-plasmon polariton condensation. As demonstrated in Appendix B, an alternative way to achieve dissipative interaction, including the near-field limit, is to facilitate the SPCM-QE interactions via a broad dark plasmon mode.

\footnotetext{
${ }^{1}$ In general, a Lindblad superoperator $\gamma \sum_{n} \hat{\mathcal{D}}_{\alpha \hat{\psi}+\beta \hat{s}_{n}^{-}}[\hat{\rho}]$ for the interacting SPCM and QE contains three independent coupling parameters $\alpha, \beta$, and $\gamma$. Here we use transformed independent parameters $\Gamma_{s p} \sim \alpha^{2} \gamma, \gamma_{\downarrow} \sim \beta^{2} \gamma$, and $\eta=\alpha \beta \gamma$.
} 


\section{NONEQUILIBRIUM PHASE DIAGRAM}

\section{A. Mean-field analysis}

The mean-field equations of motion directly follow from Eqs. (1)-(3),

$$
\begin{gathered}
\partial_{\tau} \psi=-\left(i+\bar{\Gamma}_{s p}\right) \psi-2 i \mathcal{N}_{0} \bar{\lambda} \operatorname{Re}\left[s_{-}\right]-\mathcal{N}_{0} \bar{\lambda} \bar{\eta} s_{-}, \\
\partial_{\tau} s_{-}=-\left(i+\bar{\Gamma}_{0}\right) s_{-}+4 i \bar{\lambda} s_{z} \operatorname{Re}[\psi]+2 \bar{\lambda} \bar{\eta} s_{z} \psi, \\
\partial_{\tau} s_{z}=-\bar{\gamma}_{0}\left(s_{z}-\frac{d_{0}}{2}\right)-4 \bar{\lambda} \operatorname{Im}\left[s_{-}\right] \operatorname{Re}[\psi] \\
-2 \bar{\lambda} \bar{\eta} \operatorname{Re}\left[\psi^{*} s_{-}\right],
\end{gathered}
$$

where the scalar variables are $\psi=\langle\hat{\psi}\rangle, s_{-}=\left\langle\hat{s}_{-}\right\rangle$, and $s_{z}=\left\langle\hat{s}_{z}\right\rangle$, with the normalized per site spin operators $\hat{s}_{ \pm, z}=$ $\sum_{n}\left(\hat{s}_{n}^{ \pm, z}\right) / \mathcal{N}_{0}$ and the terms in angle brackets defined as $\langle\hat{\mathcal{O}}\rangle=\operatorname{tr}(\hat{\mathcal{O}} \hat{\rho})$. In addition, $\tau=\omega_{0} t$ is a dimensionless time

$$
\mathcal{M}=\left[\begin{array}{cc}
-i-\bar{\Gamma}_{s p} & 0 \\
0 & i-\bar{\Gamma}_{s p} \\
2 s_{z} \bar{\lambda}(i+\bar{\eta}) & 2 i s_{z} \bar{\lambda} \\
-2 i s_{z} \bar{\lambda} & -2 s_{z} \bar{\lambda}(i-\bar{\eta})
\end{array}\right.
$$

To identify a phase diagram of our model as the function of dimensionless coherent $\mathcal{N}_{0} \bar{\lambda}^{2}$ and dissipative $\bar{\eta}$ coupling parameters, we look for the normal state instabilities of Eq. (7). Specifically, we calculate the eigenvalues of the matrix $\mathcal{M}$ with $s_{z}=s_{z}^{\mathrm{NS}}$ and check if at least one of them acquires a positive real part.

Figure 2 presents a phase diagram of the calculated steady states at no pumping $d_{0}=-1$ [Fig. 2(a)] and below the population inversion with $d_{0}=-0.4$ [Fig. 2(b)] with pumping where three distinct instability regions are identified. The cyan region denotes a phase associated with a single positive eigenvalue of the stability matrix that passes through zero at the boundary with the normal state. This is a signature of the pitchfork bifurcation characterizing a superradiant phase transition [27] breaking $\mathbb{Z}_{2}$ symmetry, $\psi \rightarrow-\psi$ and $s_{-} \rightarrow-s_{-}$, of Eqs. (4)-(6). Accordingly, the critical coherent coupling for this transition can be evaluated as the $\operatorname{root} \operatorname{of} \operatorname{det}[\mathcal{M}]=0$, resulting in

$$
\mathcal{N}_{0} \bar{\lambda}_{s}^{2}(\eta)=\frac{P(\bar{\eta})}{\bar{\eta}^{4} d_{0}}\left[\sqrt{1-\frac{\bar{\eta}^{4}\left(1+\bar{\Gamma}_{s p}^{2}\right)\left(1+\bar{\Gamma}_{0}^{2}\right)}{P^{2}(\bar{\eta})}}-1\right],
$$

with $P(\bar{\eta})=2+2 \bar{\eta}\left(\bar{\Gamma}_{0}+\bar{\Gamma}_{s p}\right)+\bar{\eta}^{2}\left(\bar{\Gamma}_{0} \bar{\Gamma}_{s p}-1\right)$. For vanishing dissipative coupling $\bar{\eta} \rightarrow 0$, Eq. (9) recovers a critical coupling $\mathcal{N}_{0} \bar{\lambda}_{c}^{2}=\left(1+\bar{\Gamma}_{s p}^{2}\right)\left(1+\bar{\Gamma}_{0}^{2}\right) /\left(-8 s_{z}^{\mathrm{NS}}\right)$ for the superradiant phase transition in the open Dicke model [27]. The red curves in Figs. 2(a) and 2(b) due to Eq. (9) mark well

\footnotetext{
${ }^{2}$ The linearized equation (6) for the QE inversion fluctuations $\delta s_{z}=$ $s_{z}-s_{z}^{\mathrm{NS}}$ is uncoupled from Eqs. (7) and (8) and has no effect on the normal state stability.
}

$$
\left.\begin{array}{cc}
-\mathcal{N}_{0} \bar{\lambda}(i+\bar{\eta}) & -i \mathcal{N}_{0} \bar{\lambda} \\
i \mathcal{N}_{0} \bar{\lambda} & \mathcal{N}_{0} \bar{\lambda}(i-\bar{\eta}) \\
-i-\bar{\Gamma}_{0} & 0 \\
0 & i-\bar{\Gamma}_{0}
\end{array}\right]
$$

variable and $\bar{\lambda}=\lambda / \omega_{0}(\bar{\eta}=\eta / \lambda)$ is the normalized coherent (dissipative) coupling rate. The normalized SPCM dephasing rate is $\bar{\Gamma}_{s p}=\Gamma_{s p} / \omega_{0}$ and the QE dephasing [total population decay $]$ rate is $\bar{\Gamma}_{0}=\left(\gamma_{\downarrow} / 2+\gamma_{\uparrow} / 2+\gamma_{\phi}\right) / \omega_{0}\left[\bar{\gamma}_{0}=\left(\gamma_{\downarrow}+\right.\right.$ $\left.\left.\gamma_{\uparrow}\right) / \omega_{0}\right]$. Finally, $d_{0}=\left(\gamma_{\uparrow}-\gamma_{\downarrow}\right) /\left(\gamma_{\uparrow}+\gamma_{\downarrow}\right)$ is the population inversion parameter.

The normal state is the trivial steady state of Eqs. (4)-(6) characterized by the QE population inversion $s_{z}^{\mathrm{NS}}=d_{0} / 2$ and the absence of the SPCM and QE coherences $s_{-}^{\mathrm{NS}}=\psi^{\mathrm{NS}}=$ 0 . The fluctuations of the normal state coherences $\delta \psi=\psi-$ $\psi^{\mathrm{NS}}$ and $\delta s_{-}=s_{-}-s_{-}^{\mathrm{NS}}$ satisfy the linearized equations (4) and (5) represented in the matrix form

$$
\partial_{\tau} v=\mathcal{M} \boldsymbol{v}
$$

with the vector $\boldsymbol{v}=\left[\delta \psi, \delta \psi^{*}, \delta s_{-}, \delta s_{-}^{*}\right]^{\top}$ and the stability matrix $^{2}$

the boundary between the normal state and the identified superradiant region.

The green regions in Figs. 2(a) and 2(b) mark the instabilities characterized by the simultaneous appearance of the real positive parts for two complex eigenvalues of $\mathcal{M}$. This is a signature of a critical Hopf bifurcation. In the TavisCummings model, i.e., the rotating-wave limit of the Dicke model, such an instability points to the lasing phase transition, which breaks U(1) gauge symmetry $\psi \rightarrow e^{i \bar{\omega}_{l} \tau} \psi$ and $s_{-} \rightarrow e^{i \bar{\omega}_{l} \tau} s_{-}$, where $\bar{\omega}_{l}=\omega_{l} / \omega_{0}$ is a dimensionless lasing frequency [27]. By applying the rotating-wave approximation to Eqs. (4)-(6), we recover a generalized Tavis-Cummings model (see Appendix C) with the critical parameter

$$
\begin{aligned}
\mathcal{N}_{0} \bar{\lambda}_{l}^{2}(\eta)= & \frac{\left(\bar{\eta}^{2}-1\right)\left(\bar{\Gamma}_{0}+\bar{\Gamma}_{s p}\right)^{2}}{8 \bar{\eta}^{2} d_{0}} \\
& \times\left[1 \pm \sqrt{1+\frac{16 \bar{\eta}^{2} \bar{\Gamma}_{s p} \bar{\Gamma}_{0}}{\left(\bar{\eta}^{2}-1\right)^{2}\left(\bar{\Gamma}_{0}+\bar{\Gamma}_{s p}\right)^{2}}}\right] .
\end{aligned}
$$

Such a critical parameter exists below the population inversion $-1 \leqslant d_{0}<0$ and the nonvanishing dissipative coupling if the plus (minus) sign is set in front of the square root for $0<\bar{\eta}^{2}<1\left(\bar{\eta}^{2}>1\right)$. The orange curves in Figs. 2(a) and 2(b) due to Eq. (10) mark well the boundaries between the normal state and the green regions. Accordingly, we identify the latter as lasing states without population inversion facilitated by the dissipative SPCM-QE interaction. The crossover region [gray in Figs. 2(a) and 2(b)] exhibits an interplay of both superradiant and lasing instabilities resulting in properties to be clarified below.

Above the population inversion $0<d_{0} \leqslant 1$, our generalized Dicke model recovers the regular lasing state as marked 

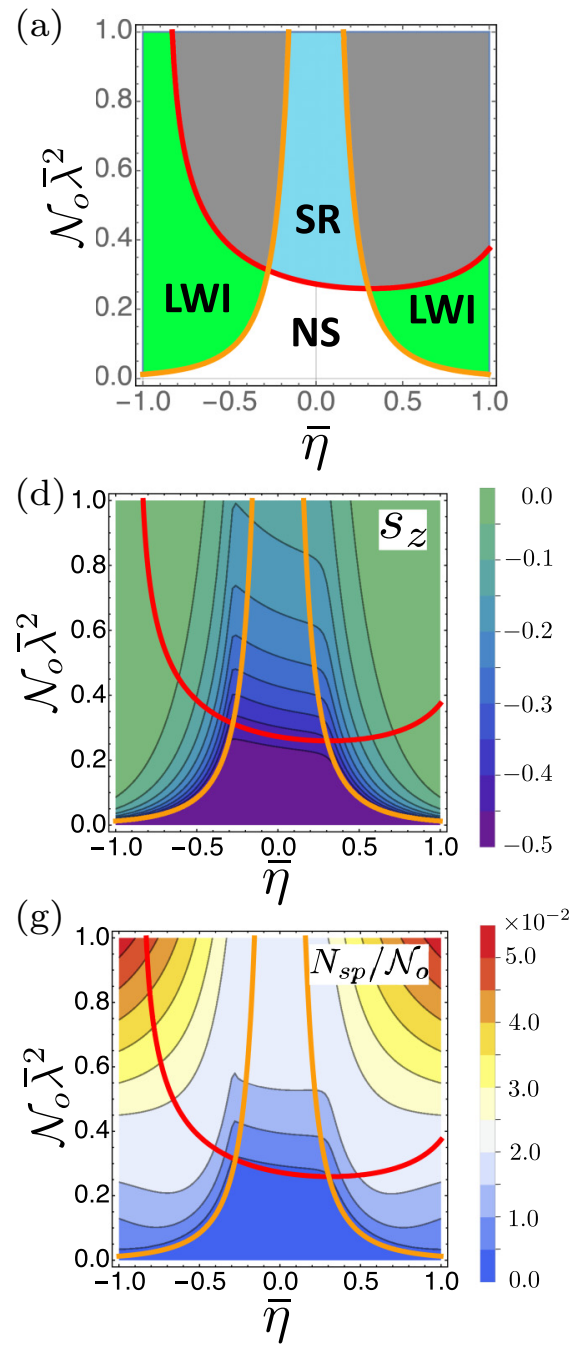
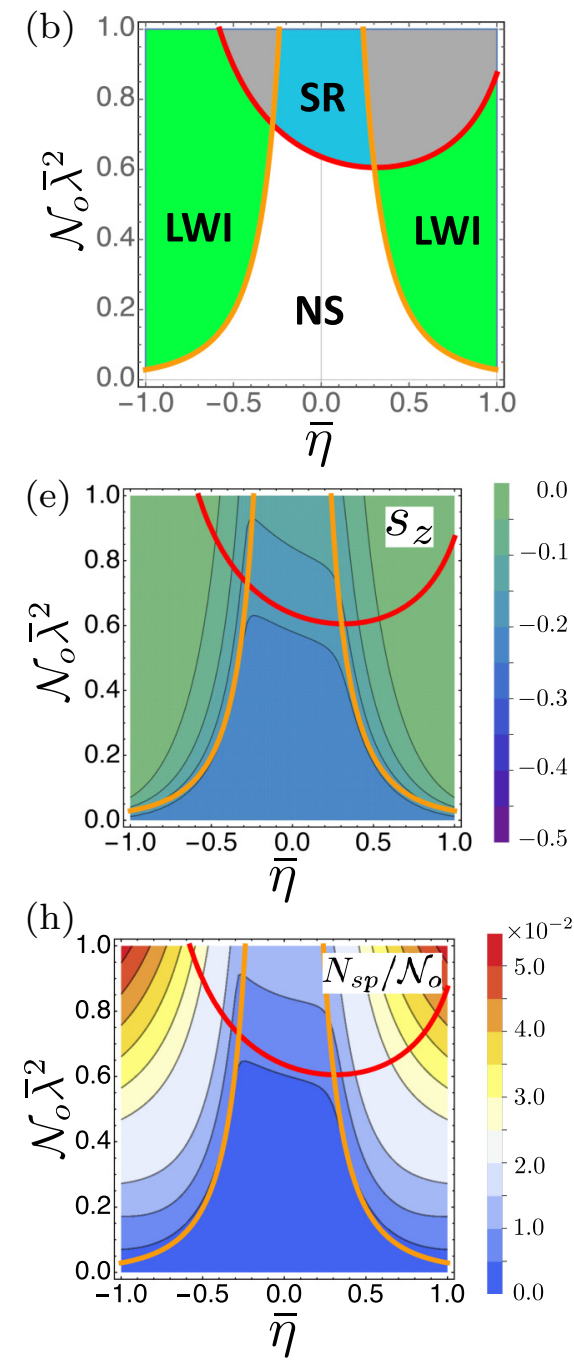
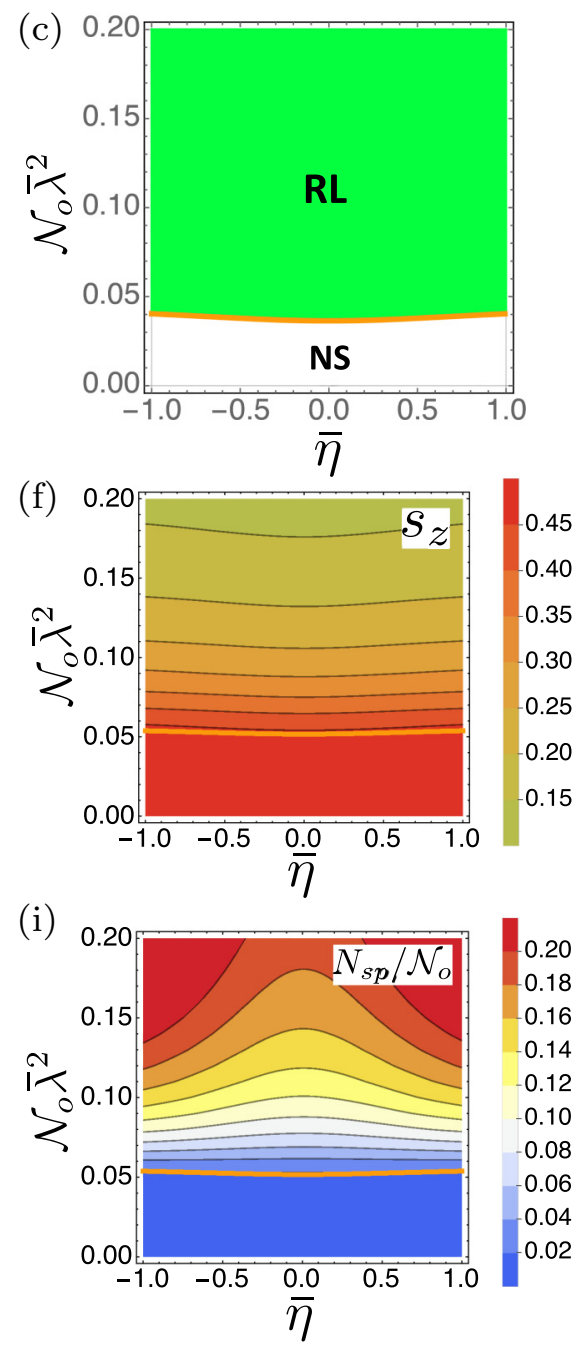

FIG. 2. (a)-(c) Mean-field phase diagrams marking the normal (NS), superradiant (SR), lasing without inversion (LWI), and regular lasing (RL) states. (d)-(f) Steady-state projection on the QE population inversion $s_{z}$ and (g)-(i) reduced SPCM population $N_{s p} / \mathcal{N}_{0}$ calculated for $\mathcal{N}_{0}=200$ using Eqs. (11)-(17). In the left, middle, and right columns $d_{0}=-1,-0.4,1$, respectively. Red [orange] curves designate $\mathcal{N}_{0} \bar{\lambda}_{s}^{2}(\eta)$ $\left[\mathcal{N}_{0} \bar{\lambda}_{l}^{2}(\eta)\right]$ boundaries. In all calculations $\bar{\Gamma}_{s p}=0.3, \gamma_{\downarrow} / \omega_{0}=0.002$, and $\gamma_{\phi} / \omega_{0}=0.02$.

in Fig. 2(c) in green. Variation of the critical boundary there is in good agreement with the predictions (orange line) of Eq. (10) where the plus (minus) sign is adopted for $\bar{\eta}^{2}>1\left(\bar{\eta}^{2}<1\right)$. In this case, a limit of $\bar{\eta} \rightarrow 0$ exists, resulting in a well-known form of the QE-cavity coupling $\mathcal{N}_{0} \bar{\lambda}_{l}^{2}=$ $\bar{\Gamma}_{0} \bar{\Gamma}_{s p} / d_{0}$ at the lasing threshold [30].

\section{B. Second-moment analysis}

To go beyond the mean-field analysis of the instability regions shown in Figs. 2(a)-2(c), we employ a set of equations of motion truncated at the level of all independent operator pairs. These averages represent a complete set of second moments of the density operator in the space of interacting QE-SPCM states. In the literature such an approximation is also known as the maximum-entropy two-particle factorization scheme [31] or the second-cumulant approximation [27,28].

Starting with Eqs. (1)-(3), we derive the following equations of motion for the SPCM population $N_{s p}=\left\langle\hat{\psi}^{\dagger} \hat{\psi}\right\rangle$ and double coherence $C_{s p}=\langle\hat{\psi} \hat{\psi}\rangle$ :

$$
\begin{aligned}
\partial_{\tau} N_{s p}= & -2 \bar{\Gamma}_{s p} N_{s p}-2 \mathcal{N}_{0} \bar{\lambda} \operatorname{Im}\left[c_{-s p}+c_{+s p}\right] \\
& -2 \mathcal{N}_{0} \bar{\lambda} \bar{\eta} \operatorname{Re}\left[c_{+s p}\right], \\
\partial_{\tau} C_{s p}= & -2\left(i+\bar{\Gamma}_{s p}\right) C_{s p}-2 i \mathcal{N}_{0} \bar{\lambda}\left[c_{-s p}+c_{+s p}\right] \\
& -2 \mathcal{N}_{0} \bar{\lambda} \bar{\eta} c_{-s p} .
\end{aligned}
$$

To close the set, equations of motion for the SPCM-QE coherences $c_{ \pm s p}=\sum_{n}\left\langle\hat{s}^{ \pm} \hat{\psi}\right\rangle / \mathcal{N}_{0}$,

$$
\begin{aligned}
\partial_{\tau} c_{-s p}=- & \left(2 i+\bar{\Gamma}_{s p}+\bar{\Gamma}_{0}\right) c_{-s p}-i \bar{\lambda}\left[\left(\mathcal{N}_{0}-1\right)\left(c_{+-}+c_{--}\right)\right. \\
- & \left.2 s_{z}\left(C_{s p}+N_{s p}\right)+1 / 2-s_{z}\right] \\
- & \bar{\lambda} \bar{\eta}\left[\left(\mathcal{N}_{0}-1\right) c_{--}-2 s_{z} C_{s p}\right], \\
\partial_{\tau} c_{+s p}= & -\left(\bar{\Gamma}_{s p}+\bar{\Gamma}_{0}\right) c_{+s p} \\
& -i \bar{\lambda}\left[\left(\mathcal{N}_{0}-1\right)\left(c_{+-}+c_{--}^{*}\right)\right. \\
& \left.+2 s_{z}\left(C_{s p}+N_{s p}\right)+1 / 2+s_{z}\right] \\
& -\bar{\lambda} \bar{\eta}\left[\left(\mathcal{N}_{0}-1\right) c_{+-}-2 s_{z} N_{s p}+1 / 2+s_{z}\right],
\end{aligned}
$$


average QE population $s_{z}$, QE double coherence $c_{--}=$ $\sum_{n \neq n^{\prime}}\left\langle\hat{s}_{n}^{-} \hat{s}_{n^{\prime}}^{-}\right\rangle / \mathcal{N}_{0}\left(\mathcal{N}_{0}-1\right)$, and intersite coherence $c_{+-}=$ $\sum_{n \neq n^{\prime}}\left\langle\hat{s}_{n}^{+} \hat{s}_{n^{\prime}}^{-}\right\rangle_{n \neq n^{\prime}} / \mathcal{N}_{0}\left(\mathcal{N}_{0}-1\right)$

$$
\begin{aligned}
\partial_{\tau} s_{z}= & -\bar{\gamma}_{0}\left(s_{z}-d_{0} / 2\right)-2 \bar{\lambda} \operatorname{Im}\left[c_{-, s p}-c_{+, s p}\right] \\
- & 2 \bar{\lambda} \bar{\eta} \operatorname{Re}\left[c_{+s p}\right], \\
\partial_{\tau} c_{--}= & -2\left(i+\bar{\Gamma}_{0}\right) c_{--}+4 i \bar{\lambda} s_{z}\left[c_{-s p}+c_{+s p}^{*}\right] \\
& +4 \bar{\lambda} \bar{\eta} s_{z} c_{-s p}, \\
\partial_{\tau} c_{+-}= & -2 \bar{\Gamma}_{0} c_{+-}-4 \bar{\lambda} s_{z} \operatorname{Im}\left[c_{+s p}-c_{-s p}\right] \\
& +4 \bar{\lambda} \bar{\eta} s_{z} \operatorname{Re}\left[c_{+s p}\right],
\end{aligned}
$$

are introduced, respectively. The $C_{s p}, c_{ \pm s p}$, and $c_{--}$are complex quantities and the associated equations should be complemented by their complex conjugates. ${ }^{3}$

In the absence of the incoherent pumping $d_{0}=-1$, Eqs. (11)-(17) describe the relaxation dynamics of the elementary excitations (introduced as initial conditions) towards the ground state whose projection on the QE population inversion $s_{z}$ and the SPCM population $N_{s p}=\left\langle\hat{\psi}^{\dagger} \hat{\psi}\right\rangle$ are shown in Figs. 2(d) and 2(g), respectively. In agreement with the mean-field calculations, the normal state is characterized by the QE ground state $s_{z}^{\mathrm{NS}}=-1 / 2$ and empty plasmonic cavity $N_{s p}=0$. However, Figs. 2(d) and 2(g) show that the presence of the counterrotating term $\hat{H}_{\mathrm{cr}}=\lambda \sum_{n}\left(\hat{\psi} \hat{s}_{n}^{-}+\hat{\psi}^{\dagger} \hat{s}_{n}^{+}\right)$in the Hamiltonian (1) results in QE and SPCM excitations as the coupling parameters cross the critical boundaries. We also observed that above the critical boundaries, the coherences appearing in Eqs. (11)-(17) become nonzero (not shown in the plot). This is an indication that the ground state associated with the symmetry breaking is a correlated state of the QEs and SPCM. Since the basis of the bare QE and SPCM states is no longer the eigenbasis, projections of the ground-state density matrix on the QE and SPCE populations show a number of virtual excitations present in the ground state. Accordingly, Eqs. (11)-(17) describe relaxation of the elementary excitations to such a correlated ground state avoiding a problem of unphysical plasmon generation in the ground state discussed in Ref. [5].

When the energy is incoherently supplied to the QEs, Figs. 2(e) and 2(f) and Figs. 2(h) and 2(i) show that, still in agreement with the mean-field theory, the normal state regions are characterized by $s_{z}^{\mathrm{NS}}=d_{0} / 2$ and $N_{s p}=0$, respectively. Observed variation of $s_{z}$ and a buildup of $N_{s p}$ within associated instability regions [Figs. 2(b) and 2(c)] indicate the nonequilibrium phase transitions to the correlated QE-SPCE states. Interestingly, the crossover region [gray in Figs. 2(a) and 2(b)] shows a clear phase boundary with the superradiant state in the associated QE and SPCM population plots. However, no signature of the phase boundary with the lasing state without inversion is seen suggesting that lasing features might be expected in this region.

\footnotetext{
${ }^{3}$ In the limit of vanishing dissipative coupling $\bar{\eta}=0$, Eqs. (11)(17) recover the results of Ref. [28].
}

\section{PHOTON EMISSION PROPERTIES}

Having established the phase diagram, we further examine photon emission spectra of the identified steady states. Keeping in mind that the SPCM emission rate exceeds the same quantity for QEs by orders of magnitude, in Appendix D we use the input-output formalism to connect the photon energy emission spectrum $S(\delta \bar{\omega})$, measured by a photodetector with the Fourier transformed SPCM operator autocorrelation function, specifically,

$$
S(\delta \bar{\omega})=(1+\delta \bar{\omega})^{4} \int_{-\infty}^{\infty} \frac{d \tau}{2 \pi}\left\langle\hat{\psi}^{\dagger}(\tau) \hat{\psi}(0)\right\rangle e^{i \delta \bar{\omega} \tau},
$$

with the dimensionless frequency detuning $\delta \bar{\omega}=(\omega-$ $\left.\omega_{0}\right) / \omega_{0}$ and the prefactor $(1+\delta \bar{\omega})^{4}$ reflecting scaling of the photon density of states and the emitted photon energy. It is noteworthy that the spectral distribution of the number of emitted photons can be obtained by normalizing $S(\delta \bar{\omega})$ per photon energy which changes the frequency prefactor to $(1+$ $\delta \bar{\omega})^{3}$. The cubic scaling of the prefactor reflects the scaling of the spontaneous decay rate determined by the vacuum photon density of states.

Applying the quantum regression theorem [30], the correlation function in Eq. (18) is calculated perturbatively via numerical solution of Eqs. (7) and (8) with $\boldsymbol{v}(\tau)=$ $\left[\langle\hat{\psi}(\tau) \hat{\psi}(0)\rangle,\left\langle\hat{\psi}^{\dagger}(\tau) \hat{\psi}(0)\right\rangle,\left\langle\hat{s}_{-}(\tau) \hat{\psi}(0)\right\rangle,\left\langle\hat{s}_{+}(\tau) \hat{\psi}(0)\right\rangle\right]^{\top}$.

The second-moment steady states are used as the initial conditions for $v(0)$ and the steady state $s_{z}$ is used to parametrize $\mathcal{M}$. According to the adopted formalism, the photon emission occurs as a result of relaxation of the SPCM-QM correlated elementary excitations to the steady/ground state accounting for virtual SPCM and QE excitations above the critical boundaries. This allows us to eliminate unphysical photon emission by such virtual states discussed in Ref. [5].

Figure 3(a) compares the emission spectra in transition from the normal to the superradiant steady state at no dissipative coupling, i.e., the $\bar{\eta}=0$ slice of the phase diagram in Fig. 2(b). Each curve has two features, one characterized by a positive detuning and the other by a negative one. The energy splitting between the features is in quantitative agreement with the spectrum of the elementary excitations [32], namely, the surface-plasmon-exciton polaritons, shown in the inset.

As the coherent coupling passes through the superradiant critical value [green dashed line in the inset in Fig. 3(a)], the lower polariton branch passes through a gap at zero photon energy $(\delta \bar{\omega}=-1)$ [33]. Emission within this spectral branch is highly suppressed due to the decrease in the photon energy and the density of states [the prefactor in Eq. (18)]. Therefore, a subtle lower polariton behavior near the critical point is not resolved in the spectra. As a result, the emission at the superradiant phase transition occurs from the upper polariton branch.

Small nonvanishing values of the incoherent coupling $\bar{\eta}$ below the threshold for the lasing without inversion result in the emission spectrum modifications shown in Figs. 3(b) and 3(c). Specifically, negative values of $\bar{\eta}$ [Fig. 3(b)] result in the emission line narrowing and strong suppression of the lower polariton peak. In contrast, positive values of $\bar{\eta}$ 

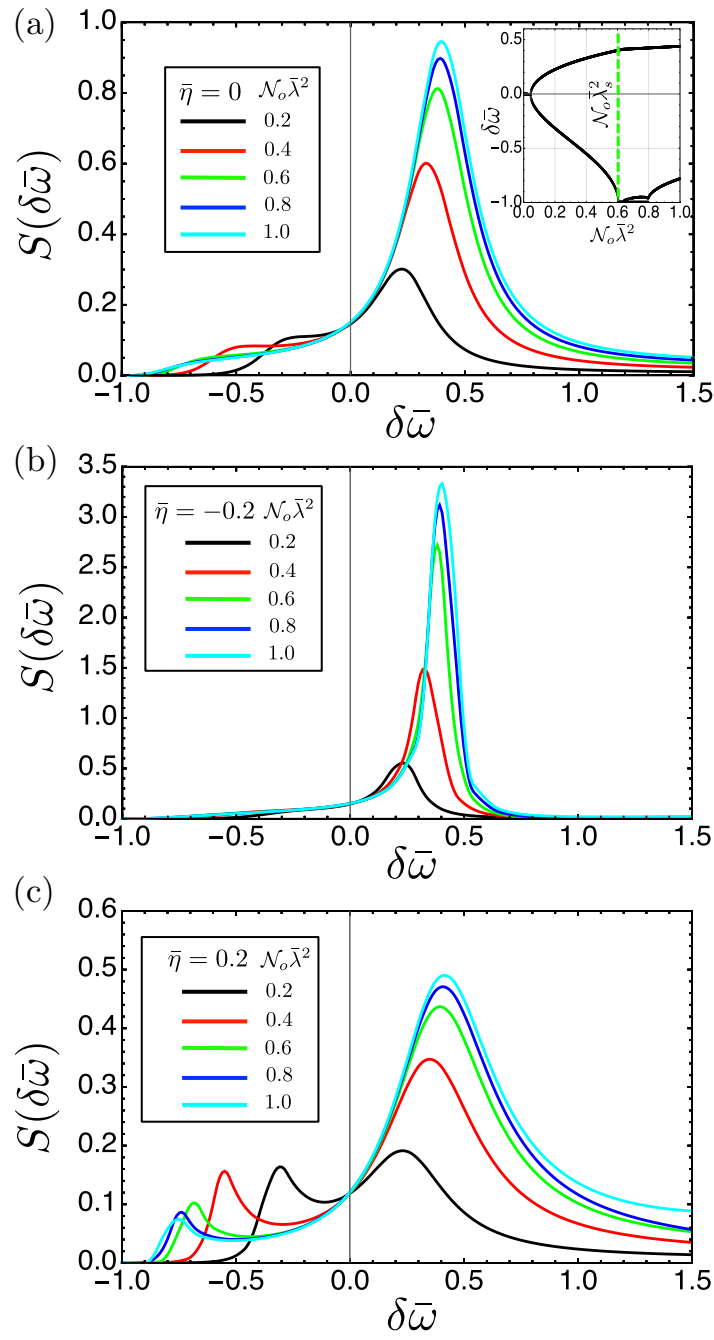

FIG. 3. Photon energy emission spectra below population inversion $d_{0}=-0.4$. The coherent coupling changes from the normal $\mathcal{N}_{0} \bar{\lambda}^{2}<0.6$ to the superradiant $\mathcal{N}_{0} \bar{\lambda}^{2} \geqslant 0.6$ region and the incoherent coupling is set to (a) $\bar{\eta}=0$, (b) $\bar{\eta}=-0.2$, and (c) $\bar{\eta}=0.2$. The inset shows the mean-field polariton spectrum for $\bar{\eta}=0$.

[Fig. 3(c)] increase the line broadening and enhance the lower polariton features.

Figure 4(a) compares emission spectra for $\bar{\eta}= \pm 0.5$ values of the dissipative coupling below (dashed line) and within (solid line) the lasing without the inversion phase shown in Fig. 2(b). Sharp characteristic features appear above the lasing critical coupling. To identify these features, we have calculated a normalized lasing frequency shift $\delta \bar{\omega}_{l}=\left(\omega_{l}-\right.$ $\left.\omega_{0}\right) / \omega_{0}$ using the generalized Tavis-Cummings model (see Appendix C)

$$
\delta \bar{\omega}_{l}(\eta)=-d_{0} \frac{2 \bar{\eta} \mathcal{N}_{0} \bar{\lambda}_{l}^{2}(\eta)}{\bar{\Gamma}_{0}+\bar{\Gamma}_{s p}},
$$

with $\mathcal{N}_{0} \bar{\lambda}_{l}^{2}(\eta)$ given by Eq. (10). According to this expression, the peak shift is determined by the dissipative coupling parameter $\bar{\eta}$.

The lasing peak shift evaluated according to Eq. (19) is plotted in the inset in Fig. 4(a). Comparison shows that the spectral positions of the sharp emission peaks are in
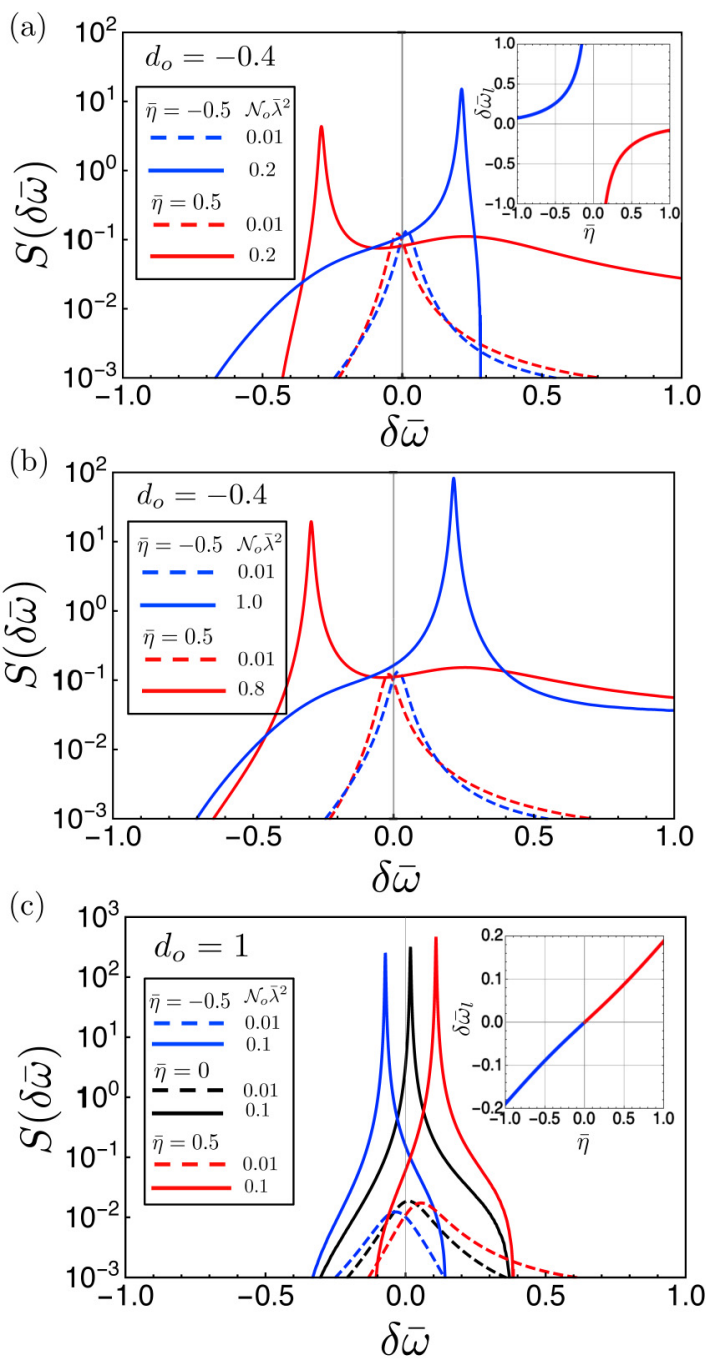

FIG. 4. Photon emission spectra. (a) and (b) Solid (dashed) lines mark lasing state without inversion (normal state) emission with the coupling threshold $\mathcal{N}_{0} \bar{\lambda}_{l}=0.2$. The inset in (a) shows the mean-field calculated lasing frequency shift. (c) Same as (a) and (b) but for the regular lasing characterized by the coupling threshold $\mathcal{N}_{0} \bar{\lambda}_{l}=0.04$.

quantitative agreement with the predictions of the TavisCummings model, allowing us to identify them as the lasing peaks. Spectra calculated within the crossover region [Fig. 4(b)] demonstrate the same trends as the spectra in Fig. 4(a). This confirms our assumption that the crossover region could demonstrate the lasing features. Finally, Fig. 4(c) compares the emission spectra for the regular lasing regime identified in Fig. 2(c). Compared to the broad spectral distribution of the photons (dashed lines) emitted below the lasing critical coupling, the spectra associated with the lasing phase show sharp monochromatic emission features. The spectral positions of the latter features are in quantitative agreement with the predictions of Eq. (19) plotted in the inset.

Comparing the insets in Figs. 4(a) and 4(c), we notice that, given the same sign of the dissipative coupling strength, the lasing frequency shifts for the lasing without inversion and regular lasing regimes show opposite signs. This trend 
resembles the behavior predicted for the counterrotating (also known as inverted) and regular lasing regimes using a generalization of the Dicke model reported in Refs. [27-29,34]. Furthermore, the counterrotating lasing occurs below the population inversion $d_{0}<0$ and has a phase boundary with the superradiant state.

However, the physical mechanisms leading to the lasing without inversion reported here and the counterrotating lasing are different. In the former case, a gain occurs as the result of the reservoir-facilitated energy flow from the SPCM to the QEs. This results in the energy supply to the QEs that overrides their losses. In the case of the counterrotating lasing, a cavity coherent emission is facilitated by the counterrotating term $\hat{H}_{\mathrm{cr}}=\lambda \sum_{n}\left(\hat{\psi} \hat{s}_{n}^{-}+\hat{\psi}^{\dagger} \hat{s}_{n}^{+}\right)$. To reach the counterrotating lasing, an imbalance between the rotating and the counterrotating terms is required. As demonstrated for atoms in a highfinesse optical cavity, such an imbalance can be engineered using coherent Raman processes due to external laser fields $[34,35]$. In contrast, the lasing without inversion predicted in this paper does not originate from the imbalanced counterrotating terms but rather emerges due to the SPCM-QM interaction facilitated by a reservoir.

\section{CONCLUSION}

Using the mean-field and second-moment analysis we identified the superradiant, lasing without inversion, and regular lasing states appearing in the phase diagram of drivendissipative plasmonic Dicke model as a result of the interplay between the coherent and dissipative SPCM-QE interaction pathways. In the limit of no dissipative coupling, we recover the results for the open Dicke and Tavis-Cummings models reported in the literature. Inclusion of the dissipative coupling extends the superradiant and regular lasing states into the dissipative coupling region and results in the emergent lasing without an inversion state. The latter stems from the incoherent energy transfer between the SPCM and the QEs. The calculated emission spectra show quantitative agreement with the predictions of the mean-field theory. The trends for the peak variations and the line-shape behavior with respect to the strengths of the dissipative coupling can be used for a spectroscopic identification of the predicted states.

\section{ACKNOWLEDGMENTS}

The work at Los Alamos National Laboratory was supported by the LANL LDRD program. A.P. also acknowledges support provided by the LANL IMS to visit Durham University and University of St. Andrews where part of this work was done. O.R. acknowledges startup funds provided by Fordham University. The work at the University of Houston was funded in part by the National Science Foundation (Grants No. CHE-1664971 and No. CHE-1836080) and the Robert A. Welch Foundation (Grant No. E-1337). E.R.B. also acknowledges the Leverhulme Trust for support at Durham University where part of this work was completed. We thank Jonathan Keeling and Peter W. Milonni for stimulating discussions.

\section{APPENDIX A: COHERENT AND DISSIPATIVE COUPLINGS FACILITATED BY THE PHOTON CONTINUUM}

Let us consider an array of QEs and metal nanoparticles shown in Fig. 1(a). Each metal nanoparticle is assigned a local surface plasmon (LSP) mode giving rise to the collective SPCM. The contribution of the dark plasmon mode is neglected in this Appendix. Further adopting the dipolar gauge [36], we treat interactions between the array components via a quantized transverse electric field, i.e., transverse photons. The Hamiltonian of such a system is

$$
\hat{H}=\hat{H}_{\mathrm{ph}}+\hat{H}_{\mathrm{SP}}+\hat{H}_{\mathrm{QE}},
$$

where the first term stands for the noninteracting photon modes

$$
\hat{H}_{\mathrm{ph}}=\sum_{\boldsymbol{q}, \lambda=1,2} \omega_{\boldsymbol{q}} \hat{a}_{\boldsymbol{q} \lambda}^{\dagger} \hat{a}_{\boldsymbol{q} \lambda}
$$

with $\hat{a}_{\boldsymbol{q} \lambda}^{\dagger}$ being a creation operator for a mode with the wave vector $\boldsymbol{q}$ and state of polarization $\lambda$. The photon frequency is $\omega_{q}=c|\boldsymbol{q}|$, with $c$ the speed of light. The second and third terms in the Hamiltonian (A1),

$$
\begin{gathered}
\hat{H}_{\mathrm{SP}}=\sum_{\bar{m}} \hbar \omega_{\bar{m}} \hat{\psi}_{\bar{m}}^{\dagger} \hat{\psi}_{\bar{m}}-\sum_{\bar{m}} \hat{\boldsymbol{p}}_{\bar{m}} \cdot \hat{\boldsymbol{E}}_{\bar{m}}^{\perp}, \\
\hat{H}_{\mathrm{QE}}=\sum_{n} \hbar \omega_{n}\left(\hat{s}_{n}^{z}+\frac{1}{2}\right)-\sum_{n} \hat{\boldsymbol{\mu}}_{n} \cdot \hat{\boldsymbol{E}}_{n}^{\perp},
\end{gathered}
$$

stand for the LSPs localized at sites $\bar{m}$ having energy $\hbar \omega_{\bar{m}}$ and described by the Bose operators $\left\{\hat{\psi}_{\bar{m}}, \hat{\psi}_{\bar{m}}^{\dagger}\right\}$ and the QEs with energies $\hbar \omega_{n}$ described by the site spin operators $\hat{s}_{n}^{\alpha}, \alpha=z, \pm$, respectively. The LSPs and QEs are coupled via transition dipole operators

$$
\begin{gathered}
\hat{\boldsymbol{p}}_{\bar{m}}=\boldsymbol{p}_{\bar{m}}\left(\hat{\psi}_{\bar{m}}^{\dagger}+\hat{\psi}_{\bar{m}}\right), \\
\hat{\boldsymbol{\mu}}_{n}=\boldsymbol{\mu}_{n}\left(\hat{s}_{n}^{+}+\hat{s}_{n}^{-}\right)
\end{gathered}
$$

to the electric fields $\hat{\boldsymbol{E}}_{\bar{m}}^{\perp} \equiv \hat{\boldsymbol{E}}^{\perp}\left(\boldsymbol{r}_{\bar{m}}\right)$ and $\hat{\boldsymbol{E}}_{n}^{\perp} \equiv \hat{\boldsymbol{E}}^{\perp}\left(\boldsymbol{r}_{n}\right)$, with $\boldsymbol{p}_{\bar{m}}$ and $\boldsymbol{\mu}_{n}$ the LSP and QE transition dipole matrix elements, respectively. The transverse electric field operator is

$$
\hat{\boldsymbol{E}}^{\perp}(\boldsymbol{r})=i \sum_{\boldsymbol{q}, \lambda=1,2} \mathcal{E}_{\boldsymbol{q} \lambda}\left(\hat{a}_{\boldsymbol{q} \lambda} e^{i \boldsymbol{q} \cdot \boldsymbol{r}}-\hat{a}_{\boldsymbol{q} \lambda}^{\dagger} e^{-i \boldsymbol{q} \cdot \boldsymbol{r}}\right),
$$

where the amplitude

$$
\mathcal{E}_{\boldsymbol{q} \lambda}=\left(\frac{\hbar \omega_{q}}{2 \varepsilon_{0} V}\right)^{1 / 2} \boldsymbol{e}_{\boldsymbol{q} \lambda}
$$

depends on the mode polarization vector $\boldsymbol{e}_{\boldsymbol{q} \lambda}$, quantization volume $V$, and vacuum permittivity $\varepsilon_{0}$.

The Heisenberg equations of motion for the LSP and QE operators due to the Hamiltonian (A1)-(A4) are

$$
\begin{gathered}
\partial_{t} \hat{\psi}_{\bar{m}}=-i \omega_{\bar{m}} \hat{\psi}_{\bar{m}}+\frac{i}{\hbar} \boldsymbol{p}_{\bar{m}} \cdot \hat{\boldsymbol{E}}_{\bar{m}}^{\perp}, \\
\partial_{t} \hat{s}_{n}^{-}=-i \omega_{n} \hat{s}_{n}^{-}-\frac{2 i}{\hbar}: \boldsymbol{\mu}_{n} \cdot \hat{\boldsymbol{E}}_{n}^{\perp} \hat{s}_{n}^{z}:, \\
\partial_{t} \hat{s}_{n}^{z}=-\frac{i}{\hbar}: \boldsymbol{\mu}_{n} \cdot \hat{\boldsymbol{E}}_{n}^{\perp}\left(\hat{s}_{n}^{-}-\hat{s}_{n}^{+}\right):,
\end{gathered}
$$


where $: \hat{A} \hat{B}$ : denotes normal ordering of the operators $\hat{A}$ and $\hat{B}$. By integrating the photon field operator equation of motion

$$
\begin{aligned}
\partial_{t} \hat{a}_{\boldsymbol{q} \lambda}= & -i \omega_{\boldsymbol{q}} \hat{a}_{\boldsymbol{q} \lambda}+\frac{1}{\hbar} \sum_{\bar{m}} \hat{\boldsymbol{p}}_{\bar{m}} \cdot \mathcal{E}_{\boldsymbol{q} \lambda} e^{-i \boldsymbol{q} \cdot \boldsymbol{r}_{\bar{m}}} \\
& +\frac{1}{\hbar} \sum_{n} \hat{\boldsymbol{\mu}}_{n} \cdot \mathcal{E}_{\boldsymbol{q} \lambda} e^{-i \boldsymbol{q} \cdot \boldsymbol{r}_{n}}
\end{aligned}
$$

and substituting the result into Eq. (A7), one partitions the electric field operator at site $\alpha=\{\bar{m}, n\}$ into three components [37]

$$
\hat{\boldsymbol{E}}_{\alpha}^{\perp}(t)=\hat{\boldsymbol{E}}_{\mathrm{in}, \alpha}^{\perp}+\hat{\boldsymbol{E}}_{\mathrm{s}, \alpha}^{\perp}+\hat{\boldsymbol{E}}_{\mathrm{rr}, \alpha}^{\perp} .
$$

The first term

$$
\begin{aligned}
\hat{\boldsymbol{E}}_{\mathrm{in}, \alpha}^{\perp}(t)= & i \sum_{\boldsymbol{q}, \lambda=1,2} \mathcal{E}_{\boldsymbol{q} \lambda}\left[\hat{a}_{\boldsymbol{q} \lambda}\left(t_{0}\right) e^{-i \omega_{q}\left(t-t_{0}\right)+i \boldsymbol{q} \cdot \boldsymbol{r}_{\alpha}}\right. \\
& \left.-\hat{a}_{\boldsymbol{q} \lambda}^{\dagger}\left(t_{0}\right) e^{i \omega_{q}\left(t-t_{0}\right)-i \boldsymbol{q} \cdot \boldsymbol{r}_{\alpha}}\right]
\end{aligned}
$$

is the input field due to the photon field initial condition at time $t_{0}$ propagated to time $t$ by the interaction free photon Hamiltonian (A2). We identify this field as the vacuum fluctuations.

The second term in Eq. (A13) describes the field produced by dipoles at their own positions

$$
\begin{aligned}
\hat{\boldsymbol{E}}_{\mathrm{rr}, \bar{m}}^{\perp} & =\lim _{r_{\bar{m}} \rightarrow 0} \stackrel{\leftrightarrow}{\mathbf{G}}\left(r_{\bar{m}}\right) \cdot \hat{\boldsymbol{p}}_{\bar{m}}\left(t-r_{\bar{m}} / c\right), \\
\hat{\boldsymbol{E}}_{\mathrm{rr}, n}^{\perp} & =\lim _{r_{n} \rightarrow 0} \stackrel{\leftrightarrow}{\mathbf{G}}\left(r_{n}\right) \cdot \hat{\boldsymbol{\mu}}_{n}\left(t-r_{n} / c\right),
\end{aligned}
$$

known as the radiation reaction field. The third term in Eq. (A13) describes the scattered field

$$
\begin{aligned}
\hat{\boldsymbol{E}}_{\mathrm{s}, \bar{m}}^{\perp}= & \sum_{\bar{m}^{\prime} \neq \bar{m}} \stackrel{\leftrightarrow}{\mathbf{G}}_{\bar{m} \bar{m}^{\prime}} \cdot \hat{\boldsymbol{p}}_{\bar{m}^{\prime}}\left(t-r_{\bar{m} \bar{m}^{\prime}} / c\right) \\
& +\sum_{n} \stackrel{\leftrightarrow}{\mathbf{G}}_{\bar{m} n} \cdot \hat{\boldsymbol{\mu}}_{n}\left(t-r_{\bar{m} n} / c\right), \\
\hat{\boldsymbol{E}}_{\mathrm{s}, n}^{\perp}= & \sum_{\bar{m}} \stackrel{\leftrightarrow}{\mathbf{G}}_{n \bar{m}} \cdot \hat{\boldsymbol{p}}_{\bar{m}}\left(t-r_{n \bar{m}} / c\right)
\end{aligned}
$$

due to the polarization of the QE and LSP dipoles surrounding either the $\bar{m}$ th LSP or the $n$th QE. Here $r_{\alpha \beta}=\left|\boldsymbol{r}_{\alpha}-\boldsymbol{r}_{\beta}\right|$ and $\alpha, \beta=\{\bar{m}, n\}$. Notice that Eq. (A18) neglects the contribution of the QEs and as a result the QE-QE interactions, justified by $p_{\bar{m}} \gg \mu_{n}$. Finally, the photon time-domain dyadic Green's function $\stackrel{\leftrightarrow}{\mathbf{G}}_{\alpha \beta}=\overleftrightarrow{\mathbf{G}}\left(r_{\alpha \beta}\right)$ appearing in Eqs. (A15)-(A18) is

$$
\begin{aligned}
\stackrel{\leftrightarrow}{\mathbf{G}}(\boldsymbol{r})= & \frac{1}{4 \pi \varepsilon_{0}}\left\{(\mathbf{n} \otimes \mathbf{n}-\stackrel{\leftrightarrow}{\mathbf{I}}) \frac{\partial_{t}^{2}}{c^{2} r}\right. \\
& \left.+(3 \mathbf{n} \otimes \mathbf{n}-\stackrel{\leftrightarrow}{\mathbf{I}})\left(\frac{\partial_{t}}{c r^{2}}+\frac{1}{r^{3}}\right)\right\},
\end{aligned}
$$

where $\stackrel{\leftrightarrow}{\mathbf{I}}$ is the unit $3 \times 3$ matrix and $\mathbf{n}=\boldsymbol{r} / r$ is a directional unit vector.

Next we use Eqs. (A9) and (A10) to eliminate the time derivatives on the right-hand side of Eqs. (A15)(A18) followed by the short-time operator expansions $\hat{\psi}_{\bar{m}^{\prime}}\left(t-r_{\alpha \bar{m}^{\prime}} / c\right) \approx \hat{\psi}_{\bar{m}^{\prime}}(t) e^{i \omega_{\bar{m}^{\prime}} r_{\alpha \bar{m}^{\prime}} / c} \quad$ and $\quad \hat{s}_{n^{\prime}}^{-}\left(t-r_{\alpha n^{\prime}} / c\right) \approx$ $\hat{s}_{n^{\prime}}^{-}(t) e^{i \omega_{n^{\prime}} r_{\alpha n^{\prime}} / c}$. This results in the radiation reaction terms describing the spontaneous radiative decay of the LSPs and QEs

$$
\begin{gathered}
\frac{i}{\hbar} \boldsymbol{p}_{\bar{m}} \cdot \hat{\boldsymbol{E}}_{\mathrm{rr}, \bar{m}}^{\perp}=-\frac{\kappa_{\bar{m}}}{2}\left(\hat{\psi}_{\bar{m}}-\hat{\psi}_{\bar{m}}^{\dagger}\right), \\
\frac{i}{\hbar} \boldsymbol{\mu}_{n} \cdot \hat{\boldsymbol{E}}_{\mathrm{rr}, n}^{\perp}=-\frac{\kappa_{n}}{2}:\left(1+\hat{\Pi}_{n}\right)\left(\hat{s}_{n}^{-}-\hat{s}_{n}^{+}\right):,
\end{gathered}
$$

with associated rates

$$
\begin{gathered}
\kappa_{\bar{m}}=\frac{p_{\bar{m}}^{2} \omega_{\bar{m}}^{3}}{3 \pi \varepsilon_{0} \hbar c^{3}}, \\
\kappa_{n}=\frac{\mu_{n}^{2} \omega_{n}^{3}}{3 \pi \varepsilon_{0} \hbar c^{3}},
\end{gathered}
$$

respectively. According to Eq. (A21), the energy transfer from QEs to LSP enhances the QE dephasing/decay rate as indicated by the polarization operator

$$
\hat{\Pi}_{n}=\sum_{\bar{m}}\left(\frac{2 \lambda_{n \bar{m}}}{\omega_{n}}\right)^{2}\left(2 \hat{\psi}_{\bar{m}}^{\dagger} \hat{\psi}_{\bar{m}}+1\right) .
$$

Evaluation of the scattered field for the LSPs and QEs gives, respectively,

$$
\begin{aligned}
\frac{i}{\hbar} \boldsymbol{p}_{\bar{m}} \cdot \hat{\boldsymbol{E}}_{\mathrm{s}, \bar{m}}^{\perp}= & -i \sum_{\bar{m}^{\prime} \neq \bar{m}} \lambda_{\bar{m} \bar{m}^{\prime}}\left(\hat{\psi}_{\bar{m}^{\prime}}+\hat{\psi}_{\bar{m}^{\prime}}^{\dagger}\right) \\
& -\sum_{\bar{m}^{\prime} \neq \bar{m}} \eta_{\bar{m} \bar{m}^{\prime}}\left(\hat{\psi}_{\bar{m}^{\prime}}-\hat{\psi}_{\bar{m}^{\prime}}^{\dagger}\right)-i \sum_{n} \lambda_{\bar{m} n}\left(\hat{s}_{n}^{-}+\hat{s}_{n}^{+}\right) \\
& -\sum_{n} \eta_{\bar{m} n}\left(\hat{s}_{n}^{-}-\hat{s}_{n}^{+}\right), \\
\frac{i}{\hbar} \boldsymbol{\mu}_{n} \cdot \hat{\boldsymbol{E}}_{\mathrm{s}, n}^{\perp}= & -i \sum_{\bar{m}} \lambda_{n \bar{m}}\left(\hat{\psi}_{\bar{m}}+\hat{\psi}_{\bar{m}}^{\dagger}\right)-\sum_{\bar{m}} \eta_{n \bar{m}}\left(\hat{\psi}_{\bar{m}}-\hat{\psi}_{\bar{m}}^{\dagger}\right) .
\end{aligned}
$$

Here the coherent $\lambda_{\alpha \beta}$ and dissipative $\eta_{\alpha \beta}$ coupling parameters naturally appear after elimination of the photon degrees of freedom and have the representation

$$
\begin{aligned}
\lambda_{\alpha \beta}= & \frac{3 d_{\alpha}}{4 d_{\beta}} \kappa_{\beta}\left[-\xi_{\alpha \beta} \frac{\cos \left(q_{\beta} r_{\alpha \beta}\right)}{q_{\beta} r_{\alpha \beta}}\right. \\
& \left.+\zeta_{\alpha \beta}\left(\frac{\sin \left(q_{\beta} r_{\alpha \beta}\right)}{\left(q_{\beta} r_{\alpha \beta}\right)^{2}}+\frac{\cos \left(q_{\beta} r_{\alpha \beta}\right)}{\left(q_{\beta} r_{\alpha \beta}\right)^{3}}\right)\right], \\
\eta_{\alpha \beta}= & \frac{3 d_{\alpha}}{4 d_{\beta}} \kappa_{\beta}\left[\xi_{\alpha \beta} \frac{\sin \left(q_{\beta} r_{\alpha \beta}\right)}{q_{\beta} r_{\alpha \beta}}\right. \\
& \left.+\zeta_{\alpha \beta}\left(\frac{\cos \left(q_{\beta} r_{\alpha \beta}\right)}{\left(q_{\beta} r_{\alpha \beta}\right)^{2}}-\frac{\sin \left(q_{\beta} r_{\alpha \beta}\right)}{\left(q_{\beta} r_{\alpha \beta}\right)^{3}}\right)\right],
\end{aligned}
$$

where

$$
\begin{aligned}
& \xi_{\alpha \beta}=\mathfrak{n}_{\alpha} \cdot \mathfrak{n}_{\beta}-\left(\mathbf{n}_{\alpha \beta} \cdot \mathfrak{n}_{\alpha}\right)\left(\mathbf{n}_{\alpha \beta} \cdot \mathfrak{n}_{\beta}\right), \\
& \zeta_{\alpha \beta}=\mathfrak{n}_{\alpha} \cdot \mathfrak{n}_{\beta}-3\left(\mathbf{n}_{\alpha \beta} \cdot \mathfrak{n}_{\alpha}\right)\left(\mathbf{n}_{\alpha \beta} \cdot \mathfrak{n}_{\beta}\right),
\end{aligned}
$$

with $\mathfrak{n}_{\alpha}=\boldsymbol{d}_{\alpha} / d_{\alpha}, \mathfrak{n}_{\beta}=\boldsymbol{d}_{\beta} / d_{\beta}, \mathbf{n}_{\alpha \beta}=\boldsymbol{r}_{\alpha \beta} / r_{\alpha \beta}, q_{\beta}=\omega_{\beta} / c$, and $r_{\alpha \beta}=\left|\boldsymbol{r}_{\alpha}-\boldsymbol{r}_{\beta}\right|$, where $\alpha, \beta=\{\bar{m}, n\}, \boldsymbol{d}_{\bar{m}}=\boldsymbol{p}_{\bar{m}}$, and $\boldsymbol{d}_{n}=$ $\boldsymbol{\mu}_{n}$. The spontaneous decay rates $\kappa_{\beta}, \beta=\{\bar{m}, n\}$, are given in Eqs. (A22) and (A23). 
The substitution of Eq. (A13) along with Eqs. (A20), (A21), (A25), and (A26) into Eqs. (A9)-(A11) results in a set of quantum Langevin equations for coupled LSP and QE operators

$$
\begin{gathered}
\partial_{t} \hat{\psi}_{\bar{m}}=-i \omega_{\bar{m}} \hat{\psi}_{\bar{m}}-\frac{\kappa_{\bar{m}}}{2}\left(\hat{\psi}_{\bar{m}}-\hat{\psi}_{\bar{m}}^{\dagger}\right)-i \sum_{\bar{m}^{\prime} \neq \bar{m}} \lambda_{\bar{m} \bar{m}^{\prime}}\left(\hat{\psi}_{\bar{m}^{\prime}}+\hat{\psi}_{\bar{m}^{\prime}}^{\dagger}\right)-\sum_{\bar{m}^{\prime} \neq \bar{m}} \eta_{\bar{m}_{\bar{m}}^{\prime}}\left(\hat{\psi}_{\bar{m}^{\prime}}-\hat{\psi}_{\bar{m}^{\prime}}^{\dagger}\right)-i \sum_{n} \lambda_{\bar{m} n}\left(\hat{s}_{n}^{-}+\hat{s}_{n}^{+}\right) \\
\quad-\sum_{n} \eta_{\bar{m} n}\left(\hat{s}_{n}^{-}-\hat{s}_{n}^{+}\right)+\frac{i}{\hbar} \boldsymbol{p}_{\bar{m}} \cdot \hat{\boldsymbol{E}}_{\mathrm{in}, \bar{m}}^{\perp}, \\
\partial_{t} \hat{s}_{n}^{-}=-i \omega_{n} \hat{s}_{n}^{-}-\frac{\kappa_{n}}{2}:\left(1+\hat{\Pi}_{n}\right)\left(\hat{s}_{n}^{-}-\hat{s}_{n}^{+}\right):+2 i \sum_{\bar{m}} \lambda_{n \bar{m}}: \hat{s}_{n}^{z}\left(\hat{\psi}_{\bar{m}}+\hat{\psi}_{\bar{m}}^{\dagger}\right):+2 \sum_{\bar{m}} \eta_{n \bar{m}}: \hat{s}_{n}^{z}\left(\hat{\psi}_{\bar{m}}-\hat{\psi}_{\bar{m}}^{\dagger}\right):-\frac{2 i}{\hbar}: \boldsymbol{\mu}_{n} \cdot \hat{\boldsymbol{E}}_{\mathrm{in}, n}^{\perp} \hat{s}_{n}^{z}: \quad(\mathrm{A} 32) \\
\partial_{t} \hat{s}_{n}^{z}=-\kappa_{n}\left(1+\hat{\Pi}_{n}\right)\left(\hat{s}_{n}^{z}+\frac{1}{2}\right)+i \sum_{\bar{m}} \lambda_{n \bar{m}}:\left(\hat{s}_{n}^{-}-\hat{s}_{n}^{+}\right)\left(\hat{\psi}_{\bar{m}}+\hat{\psi}_{\bar{m}}^{\dagger}\right):+\sum_{\bar{m}} \eta_{n \bar{m}}:\left(\hat{s}_{n}^{-}-\hat{s}_{n}^{+}\right)\left(\hat{\psi}_{\bar{m}}-\hat{\psi}_{\bar{m}}^{\dagger}\right):-\frac{i}{\hbar}: \boldsymbol{\mu}_{n} \cdot \hat{\boldsymbol{E}}_{\mathrm{in}, n}^{\perp}\left(\hat{s}_{n}^{-}-\hat{s}_{n}^{+}\right):
\end{gathered}
$$

According to Eqs. (A31)-(A33), the photon continuum fluctuations, i.e., $\hat{\boldsymbol{E}}_{\mathrm{in}}^{\perp}$, result in cooperative dissipation processes such as spontaneous radiative decay of LSPs including the superradiant emission due to the LSP-LSP coupling with the rate $\eta_{\bar{m} \bar{m}^{\prime}}$, the QE radiative decay, and the LSP-QE dissipative coupling with the rate $\eta_{n \bar{m}}$.

The Dicke model assumes identical LSPs (QEs), frequencies $\omega_{\bar{m}}=\omega_{s p}\left(\omega_{n}=\omega_{0}\right)$, and transition dipoles $\boldsymbol{p}_{\bar{m}}=\boldsymbol{p}\left(\boldsymbol{\mu}_{n}=\boldsymbol{\mu}\right)$ corresponding to identical spontaneous radiative decay rates $\kappa_{\bar{m}}=\kappa_{s p}\left(\kappa_{n}=\kappa_{0}\right)$. Furthermore, we introduce collective LSP modes characterized by momentum $k$,

$$
\hat{\psi}_{k}=\frac{1}{\sqrt{\overline{\mathcal{N}}}} \sum_{\bar{m}=1}^{\overline{\mathcal{N}}} \hat{\psi}_{\bar{m}} e^{-i k \bar{m}},
$$

where $\overline{\mathcal{N}}$ is the number of metal nanoparticles in the array. Identifying the SPCM as the $k=0$ collective mode, $\hat{\psi} \equiv \hat{\psi}_{k=0}$, we simplify Eqs. (A31)-(A33) to the form

$$
\begin{gathered}
\partial_{t} \hat{\psi}=-i\left(\omega_{s p}+\lambda_{s p}\right) \hat{\psi}-\frac{1}{2}\left(\kappa_{s p}+\eta_{s p}\right)\left(\hat{\psi}-\hat{\psi}^{\dagger}\right)-i \lambda \sum_{n}\left(\hat{s}_{n}^{-}+\hat{s}_{n}^{+}\right)-\eta \sum_{n}\left(\hat{s}_{n}^{-}-\hat{s}_{n}^{+}\right)+\frac{i}{\hbar} \boldsymbol{p} \cdot \hat{\boldsymbol{E}}_{\mathrm{in}, s p}^{\perp} \\
\partial_{t} \hat{s}_{n}^{-}=-i \omega_{0} \hat{s}_{n}^{-}-\frac{1}{2}\left[\kappa_{0}+\gamma_{s p} \hat{\psi}^{\dagger} \hat{\psi}\right]\left(\hat{s}_{n}^{-}-\hat{s}_{n}^{+}\right)+2 i \lambda: \hat{s}_{n}^{z}\left(\hat{\psi}+\hat{\psi}^{\dagger}\right):+2 \eta: \hat{s}_{n}^{z}\left(\hat{\psi}-\hat{\psi}^{\dagger}\right):-\frac{2 i}{\hbar}: \boldsymbol{\mu} \cdot \hat{\boldsymbol{E}}_{\mathrm{in}, n}^{\perp} \hat{s}_{n}^{z}: \\
\partial_{t} \hat{s}_{n}^{z}=-\left[\kappa_{0}+\gamma_{s p} \hat{\psi}^{\dagger} \hat{\psi}\right]\left(\hat{s}_{n}^{z}+\frac{1}{2}\right)+i \lambda:\left(\hat{s}_{n}^{-}-\hat{s}_{n}^{+}\right)\left(\hat{\psi}+\hat{\psi}^{\dagger}\right):+\eta:\left(\hat{s}_{n}^{-}-\hat{s}_{n}^{+}\right)\left(\hat{\psi}-\hat{\psi}^{\dagger}\right):-\frac{i}{\hbar}: \boldsymbol{\mu} \cdot \hat{\boldsymbol{E}}_{\mathrm{in}, n}^{\perp}\left(\hat{s}_{n}^{-}-\hat{s}_{n}^{+}\right):
\end{gathered}
$$

where

$$
\hat{\boldsymbol{E}}_{\mathrm{in}, s p}^{\perp}=\frac{1}{\sqrt{\overline{\mathcal{N}}}} \sum_{\bar{m}=1}^{\overline{\mathcal{N}}} \hat{\boldsymbol{E}}_{\mathrm{in}, \bar{m}}^{\perp} .
$$

In Eqs. (A35)-(A37), the SPCM band edge renormalization, superradiant decay, and the QE plasmon-assisted spontaneous decay rates are

$$
\begin{aligned}
& \lambda_{s p}=\frac{1}{\overline{\mathcal{N}}} \sum_{\bar{m}=1}^{\overline{\mathcal{N}}-1} \lambda_{\bar{m} \overline{\mathcal{N}}}, \\
& \eta_{s p}=\frac{2}{\overline{\mathcal{N}}} \sum_{\bar{m}=1}^{\overline{\mathcal{N}}-1} \eta_{\bar{m} \overline{\mathcal{N}}}, \\
& \gamma_{s p}=2 \overline{\mathcal{N}} \kappa_{s p}\left(\frac{\lambda}{\omega_{0}}\right)^{2},
\end{aligned}
$$

respectively. The SPCM-QE coherent $\lambda$ and dissipative $\eta$ coupling rates are, respectively,

$$
\begin{aligned}
& \lambda=\frac{1}{\sqrt{\overline{\mathcal{N}}}} \sum_{\bar{m}=1}^{\overline{\mathcal{N}}} \lambda_{n \bar{m}}, \\
& \eta=\frac{1}{\sqrt{\overline{\mathcal{N}}}} \sum_{\bar{m}=1}^{\overline{\mathcal{N}}} \eta_{n \bar{m}} .
\end{aligned}
$$

Above, we also assumed that the QEs are arranged so that each has coherent and dissipative coupling rates identical to those of the SPCM.

The model represented by Eqs. (A35)-(A43) recovers the generalized Dicke model introduced in Sec. II, provided the counterrotating dissipative terms and the plasmon-assisted QE decay operator, i.e., $\gamma_{s p} \hat{\psi}^{\dagger} \hat{\psi}$, are dropped and the SPCM and $\mathrm{QE}$ nonradiative decay and dephasing rates are added.

\section{APPENDIX B: TWO-MODE SURFACE-PLASMON MODEL ALLOWING FOR DISSIPATIVE COUPLING}

The Dicke Hamiltonian accounting for two surfaceplasmon modes coupled to the QEs and with each other reads

$$
\begin{aligned}
\hat{H}= & \Omega \hat{\phi}^{\dagger} \hat{\phi}+\omega_{0} \hat{\psi}^{\dagger} \hat{\psi}+\omega_{0}\left(\sum_{n} \hat{s}_{n}^{z}+\frac{\mathcal{N}_{0}}{2}\right) \\
& +\zeta \hat{Q} \hat{q}+2(\xi \hat{Q}+\lambda \hat{q}) \sum_{n} \hat{s}_{n}^{x}
\end{aligned}
$$

Here and below, the quadrature representation $\hat{Q}=\hat{\phi}+\hat{\phi}^{\dagger}$, $\hat{q}=\hat{\psi}+\hat{\psi}^{\dagger}, \quad \hat{s}_{n}^{x}=\left(s_{n}^{+}+\hat{s}_{n}^{-}\right) / 2$, and $\hat{s}_{n}^{y}=i\left(s_{n}^{-}-\hat{s}_{n}^{+}\right) / 2$ is used for the sake of brevity. The coupling parameters $\zeta, \xi$, 
and $\lambda$ can be obtained microscopically in the dipole-dipole approximation using an approach developed in Ref. [14].

A set of the Heisenberg equations of motion due to the Hamiltonian (B1) reads

$$
\begin{gathered}
\partial_{t} \hat{\phi}=-(i \Omega+\Lambda) \hat{\phi}-i \zeta \hat{q}-2 i \xi \sum_{n} \hat{s}_{n}^{x}, \\
\partial_{t} \hat{\psi}=-\left(i \omega_{0}+\Gamma_{s p}\right) \hat{\psi}-i \zeta \hat{Q}-2 i \lambda \sum_{n} \hat{s}_{n}^{x}, \\
\partial_{t} \hat{s}_{n}^{-}=-\left(i \omega_{0}+\Gamma_{0}\right) \hat{s}_{n}^{-}+2 i \hat{s}_{n}^{z}(\xi \hat{Q}+\lambda \hat{q}), \\
\partial_{t} \hat{s}_{n}^{z}=-\gamma_{0}\left(\hat{s}_{n}^{z}-d_{0} / 2\right)+2 \hat{s}_{n}^{y}(\xi \hat{Q}+\lambda \hat{q}),
\end{gathered}
$$

where the SPCM dephasing rate $\Gamma_{s p}$, the QE dephasing $\Gamma_{0}=$ $\left(\gamma_{\downarrow} / 2+\gamma_{\uparrow} / 2+\gamma_{\phi}\right)$ and population decay $\gamma_{0}=\gamma_{\downarrow}+\gamma_{\uparrow}$ rates, and the inversion parameter $d_{0}=\left(\gamma_{\uparrow}-\gamma_{\downarrow}\right) /\left(\gamma_{\uparrow}+\gamma_{\downarrow}\right)$ are added as discussed in Sec. II.

Integrating Eq. (B2) in the Markovian approximation and neglecting the counterrotating terms containing $e^{i\left(\omega_{0}+\Omega\right) t^{\prime}}$ gives

$$
\begin{aligned}
\hat{\phi}(t)= & \hat{\phi}(0) e^{-(i \Omega+\Lambda) t} \\
& -\frac{\Delta+i \Lambda}{\Delta^{2}+\Lambda^{2}}\left[\zeta \hat{\psi}(t)+\xi \sum_{n} \hat{s}_{n}^{-}(t)\right],
\end{aligned}
$$

with the detuning $\Delta=\Omega-\omega_{0}$. Taking into account that the dark plasmon mode is broad $\Delta \ll \Lambda$, we can safely set $\Delta=0$ in Eq. (B6) and neglect the contribution of the initial condition $\hat{\phi}(0)$ which should decay at time $t$.

By substituting Eq. (B6) with $\Delta=0$ and $\hat{\phi}(0)=0$ into Eqs. (B3)-(B5), we obtain our final set of operator equations where the dark plasmon mode is integrated out:

$$
\begin{gathered}
\partial_{t} \hat{\psi}=-\left(i \omega_{0}+\gamma_{s p}\right) \hat{\psi}-\eta_{s p}\left(\hat{\psi}-\hat{\psi}^{\dagger}\right)-i \lambda \sum_{n}\left(\hat{s}_{n}^{-}+\hat{s}_{n}^{+}\right)-i \eta \sum_{n}\left(\hat{s}_{n}^{-}-\hat{s}_{n}^{+}\right), \\
\partial_{t} \hat{s}_{n}^{-}=-\left(i \omega_{0}+\gamma_{0}\right) \hat{s}_{n}^{-}-\eta_{\mathrm{QE}}\left(\hat{s}_{n}^{-}-\hat{s}_{n}^{+}\right)+2 \eta_{\mathrm{QE}}: s_{n}^{z} \sum_{m \neq n}\left(\hat{s}_{m}^{-}-\hat{s}_{m}^{+}\right):+i \lambda: \hat{s}_{n}^{z}\left(\hat{\psi}+\hat{\psi}^{\dagger}\right):-\eta: \hat{s}_{n}^{z}\left(\hat{\psi}-\hat{\psi}^{\dagger}\right): \\
\partial_{t} \hat{s}_{n}^{z}=-\gamma_{t}\left(\hat{s}_{n}^{z}-\frac{d_{0}}{2}\right)-2 \eta_{\mathrm{QE}}\left(\hat{s}_{n}^{z}+\frac{1}{2}\right)+\eta_{\mathrm{QE}}:\left(\hat{s}_{n}^{-}-\hat{s}_{n}^{+}\right) \sum_{m \neq n}\left(\hat{s}_{m}^{-}-\hat{s}_{m}^{+}\right):+i \lambda:\left(\hat{s}_{m}^{-}-\hat{s}_{m}^{+}\right)\left(\hat{\psi}+\psi \hat{\psi}^{\dagger}\right):+:\left(\hat{s}_{n}^{-}-\hat{s}_{n}^{+}\right)\left(\hat{\psi}^{+}+\psi^{\dagger}\right): .
\end{gathered}
$$

Here $: \hat{A} \hat{B}$ : denotes normal ordering of the operators $\hat{A}$ and $\hat{B}$. Due to the dark plasmon mode Eqs. (B7)-(B9) acquire the dissipative rates $\eta_{s p}=\zeta^{2} / \Lambda, \eta_{\mathrm{QE}}=\xi^{2} / \Lambda$, and $\eta=\xi \zeta / \Lambda$ describing the SPCM decay, QEs cooperative decay, and SPCM-QE dissipative coupling, respectively.

The generalized Dicke model in Sec. II can be recovered from Eqs. (B7)-(B9) by neglecting the terms $2 \eta_{\mathrm{QE}}: s_{n}^{z} \sum_{m \neq n}\left(\hat{s}_{m}^{-}-\hat{s}_{m}^{+}\right)$: and $\eta_{\mathrm{QE}}:\left(\hat{s}_{n}^{-}-\hat{s}_{n}^{+}\right) \sum_{m \neq n}\left(\hat{s}_{m}^{-}-\hat{s}_{m}^{+}\right)$: and dropping the counterrotating dissipative terms.

\section{APPENDIX C: GENERALIZED TAVIS-CUMMINGS MODEL}

We generalize a driven-dissipative Tavis-Cummings model by including the dissipative coupling between the SPCM and QEs. Associated equations of motion

$$
\begin{gathered}
\partial_{\tau} \psi=-\left(i+\bar{\Gamma}_{s p}\right) \psi-\bar{\lambda}(i+\bar{\eta}) \mathcal{N}_{0} s_{-}, \\
\partial_{\tau} s_{-}=-\left(i+\bar{\Gamma}_{0}\right) s_{-}+2 \bar{\lambda}(i+\bar{\eta}) s_{z} \psi, \\
\partial_{\tau} s_{z}=-\bar{\gamma}_{0}\left(s_{z}-d_{0} / 2\right)+2 \bar{\lambda} \operatorname{Re}\left[(i-\bar{\eta}) \psi^{*} s_{-}\right]
\end{gathered}
$$

result from Eqs. (4)-(6) after applying the rotating-wave approximation.

We further break the U(1) gauge symmetry of Eqs. (C1)(C3) by setting the phase $\psi \rightarrow e^{i \bar{\omega}_{l} \tau} \psi$ and $s_{-} \rightarrow e^{i \bar{\omega}_{l} \tau} s_{-}$ with $\bar{\omega}_{l}=\omega_{l} / \omega_{0}$ to be identified as the normalized lasing frequency. As a result, the equations of motion for the coherences become

$$
\partial_{\tau} \psi=-\left(i \delta \bar{\omega}_{l}+\bar{\Gamma}_{s p}\right) \psi-\bar{\lambda}(i+\bar{\eta}) \mathcal{N}_{0} s_{-},
$$

$$
\partial_{\tau} s_{-}=-\left(i \delta \bar{\omega}_{l}+\bar{\Gamma}_{0}\right) s_{-}+2 \bar{\lambda}(i+\bar{\eta}) s_{z} \psi
$$

where $\delta \bar{\omega}_{l}=1-\bar{\omega}_{l}$ defines a normalized frequency detuning. Equation (C3) does not change under such a transformation. Using the steady-state solution of Eq. (C5),

$$
s_{-}=\frac{2 \bar{\lambda}(i+\bar{\eta})}{i \delta \bar{\omega}_{l}+\bar{\Gamma}_{0}} s_{z} \psi,
$$

we eliminate $s_{-}$in Eq. (C4) and in the steady-state solution of Eq. (C3) to obtain

$$
\begin{gathered}
\partial_{\tau} \psi=-\left[\left(i \delta \bar{\omega}_{l}+\bar{\Gamma}_{s p}\right)+2 \bar{\lambda}^{2} \mathcal{N}_{0} \frac{(i+\bar{\eta})^{2}}{i \delta \bar{\omega}_{l}+\bar{\Gamma}_{0}} s_{z}\right] \psi, \\
s_{z}=\frac{d_{0}}{2}\left[1+4 \bar{\lambda}^{2} \frac{\bar{\Gamma}_{0}}{\bar{\gamma}_{0}} \frac{1+\bar{\eta}^{2}}{\delta \bar{\omega}_{l}^{2}+\bar{\Gamma}_{0}^{2}}|\psi|^{2}\right]^{-1} .
\end{gathered}
$$

The lasing threshold can be found by equating to zero both the real and imaginary parts of the expression in the square brackets of Eq. (C7). The roots of these two equation with respect to $\delta \bar{\omega}_{l}$ and $s_{z}$ stand for the lasing frequency detuning $\delta \bar{\omega}_{l}(\bar{\eta})[$ Eq. (19)] and the expression for the steady-state population inversion

$$
s_{z}=\frac{d_{0}}{2} \frac{\bar{\lambda}_{l}^{2}(\bar{\eta})}{\mathcal{N}_{0} \bar{\lambda}^{2}} .
$$

Here the critical coupling $\bar{\lambda}_{l}^{2}(\bar{\eta})$ is defined by Eq. (10) and the expression is valid for $\bar{\lambda}_{l}^{2}(\bar{\eta}) \leqslant \bar{\lambda}^{2}$. Finally, making the substitution of Eq. (C9) into Eq. (C8), we find the order 
parameter

$$
|\psi|^{2}=\frac{\bar{\gamma}_{0}\left[\delta \bar{\omega}_{l}^{2}(\bar{\eta})+\bar{\Gamma}_{0}^{2}\right]}{4 \bar{\Gamma}_{0} \bar{\lambda}_{l}^{2}(\bar{\eta})\left(1+\bar{\eta}^{2}\right)}\left(1-\frac{\bar{\lambda}_{l}^{2}(\bar{\eta})}{\mathcal{N}_{0} \bar{\lambda}^{2}}\right),
$$

representing the SPCM spontaneous coherence for $\bar{\lambda}^{2} \geqslant$ $\bar{\lambda}_{l}^{2}(\bar{\eta})$. The expression for the QE spontaneous coherence

$$
\left|s_{-}\right|^{2}=\frac{\bar{\gamma}_{0} \bar{\lambda}_{l}^{2}(\bar{\eta})}{\bar{\Gamma}_{0} \mathcal{N}_{0} \bar{\lambda}^{2}}\left(1-\frac{\bar{\lambda}_{l}^{2}(\bar{\eta})}{\mathcal{N}_{0} \bar{\lambda}^{2}}\right)
$$

follows from Eqs. (C6) and (C10).

\section{APPENDIX D: RELATIONSHIP BETWEEN OUTPUT ELECTRIC FIELD AND PHOTON EMISSION ENERGY SPECTRUM}

Taking into account that the surface-plasmon transition dipole exceeds the QE ones, i.e., $p_{s p} \gg \mu_{n}$, the cavity emission is mostly contributed by the SPCM, which indeed can be strongly coupled to the QEs. To find an output electric field produced by the cavity in the far-field photodetector zone, we employ the Heisenberg equation of motion (A12) for the photon mode operator in which the right-hand side QE term is dropped by setting $\mu_{n}=0$.

Following the input output formalism $[30,38]$, we integrate Eq. (A12) both forward $\left(t>t_{0}\right)$ and backward $\left(t_{f}>t\right)$ in time. This provides us with the expression for the cavity output field

$$
\hat{\boldsymbol{E}}_{\mathrm{out}}^{\perp}(\boldsymbol{r}, t)=\hat{\boldsymbol{E}}_{\mathrm{in}}^{\perp}(\boldsymbol{r}, t)+\sum_{\bar{m}} \stackrel{\leftrightarrow}{\mathbf{G}}\left(\boldsymbol{r}-\boldsymbol{r}_{\bar{m}}\right) \cdot \hat{\boldsymbol{p}}_{\bar{m}}\left(t-\left|\boldsymbol{r}-\boldsymbol{r}_{\bar{m}}\right| / c\right)
$$

in terms of the input field (A14) and the LSP transition dipole operator $\hat{\boldsymbol{p}}_{\bar{m}}$ (A5) propagated by the dyadic photon Green's function (A19) to the detector at coordinate $\boldsymbol{r}$.

Since the cavity-detector separation significantly exceeds the cavity linear size and $t \gg\left|\boldsymbol{r}-\boldsymbol{r}_{\bar{m}}\right| / c$, we can replace the LSP mode with the collective SPCM (A34) and retain only the far-field term in the photon Green's function. Finally, partitioning the output field into the negative and positive frequency components $\hat{\boldsymbol{E}}_{\text {out }}^{\perp}=\hat{\boldsymbol{E}}_{\text {out }}^{-}+\hat{\boldsymbol{E}}_{\text {out }}^{+}$[39], we obtain

$$
\begin{aligned}
& \hat{\boldsymbol{E}}_{\text {out }}^{-}(\boldsymbol{r}, t)=\hat{\boldsymbol{E}}_{\mathrm{in}}^{-}(\boldsymbol{r}, t)-\frac{\boldsymbol{p}_{s p}-\mathbf{n}\left(\mathbf{n} \cdot \boldsymbol{p}_{s p}\right)}{4 \pi \varepsilon_{0} c^{2} r} \partial_{t}^{2} \hat{\psi}^{\dagger}(t), \\
& \hat{\boldsymbol{E}}_{\mathrm{out}}^{+}(\boldsymbol{r}, t)=\hat{\boldsymbol{E}}_{\mathrm{in}}^{+}(\boldsymbol{r}, t)-\frac{\boldsymbol{p}_{s p}-\mathbf{n}\left(\mathbf{n} \cdot \boldsymbol{p}_{s p}\right)}{4 \pi \varepsilon_{0} c^{2} r} \partial_{t}^{2} \hat{\psi}(t),
\end{aligned}
$$

where $\boldsymbol{r}$ denotes the radius vector from the cavity and a detector, $\mathbf{n}=\boldsymbol{r} / r$ with $r=|\boldsymbol{r}|$, and $\hat{\boldsymbol{E}}_{\text {in }}^{-}=\left(\hat{\boldsymbol{E}}_{\text {in }}^{+}\right)^{\dagger}$ is due to the first term on the right-hand side of Eq. (A14).

The power emitted by the cavity is

$$
\frac{d E}{d t}=2 \varepsilon_{0} c r^{2} \int_{4 \pi^{2}} d \Omega\left\langle\hat{\boldsymbol{E}}_{\text {out }}^{-}(\boldsymbol{r}, t) \cdot \hat{\boldsymbol{E}}_{\text {out }}^{+}(\boldsymbol{r}, t)\right\rangle,
$$

where the integration is perform over the whole solid angle surrounding the cavity. The substitution of Eqs. (D2) and (D3) into Eq. (D4) and subsequent integration over the solid angle results in

$$
\frac{d E(t)}{d t}=\frac{p_{s p}^{2}}{3 \pi \varepsilon_{0} c^{3}}\left\langle\partial_{t}^{2} \hat{\psi}^{\dagger}(t) \partial_{t}^{2} \hat{\psi}(t)\right\rangle .
$$

The correlation functions containing the input field fluctuations vanish after averaging.

Following the Wiener-Khintchine theorem [40], we define the photon power spectrum in terms of the SPCM operator autocorrelation function as

$$
\begin{aligned}
S(\omega) \delta\left(\omega-\omega^{\prime}\right)= & \int_{-\infty}^{\infty} \frac{d t}{2 \pi} \int_{-\infty}^{\infty} \frac{d t^{\prime}}{2 \pi} \\
& \times\left\langle\partial_{t}^{2} \hat{\psi}^{\dagger}(t) \partial_{t}^{2} \hat{\psi}\left(t^{\prime}\right)\right\rangle e^{i \omega t-i \omega^{\prime} t},
\end{aligned}
$$

which simplifies to the form

$$
\begin{aligned}
S(\omega) \delta\left(\omega-\omega^{\prime}\right)= & \omega^{2} \omega^{2} \int_{-\infty}^{\infty} \frac{d t}{2 \pi} \int_{-\infty}^{\infty} \frac{d t^{\prime}}{2 \pi} \\
& \times\left\langle\hat{\psi}^{\dagger}(t) \hat{\psi}\left(t^{\prime}\right)\right\rangle e^{i \omega t-i \omega^{\prime} t^{\prime}} .
\end{aligned}
$$

For the stationary process, one integral on the right-hand side of Eq. (D7) can be evaluated, resulting in $\delta\left(\bar{\omega}-\bar{\omega}^{\prime}\right)$, to recover the form of Eq. (18) for $S(\omega)$. According to Eqs. (D5)(D7), the photon energy emitted by the cavity per frequency range $d \omega$ is related to the spectral function $S(\omega)$ as

$$
\frac{d E(\omega)}{d \omega}=\frac{p_{s p}^{2}}{3 \pi \varepsilon_{0} c^{3}} S(\omega) .
$$

Note that an expression for the (power) spectrum of the photons emitted within an infinitesimally small solid angle $d \Omega$ can be obtained by simply multiplying [Eq. (D5)] Eq. (D8) with the prefactor $3 \sin ^{2} \theta / 8 \pi$ where the angle $\theta$ is measured between $\boldsymbol{p}_{s p}$ and the radius vector pointing to the detector.
[1] M. Tame, K. McEnery, Ş. Özdemir, J. Lee, S. Maier, and M. Kim, Quantum plasmonics, Nat. Phys. 9, 329 (2013).

[2] J. A. Hollingsworth, H. Htoon, A. Piryatinski, S. Götzinger, and V. Sandoghdar, When excitons and plasmons meet: Emerging function through synthesis and assembly, MRS Bull. 40, 768 (2015).

[3] M. Sukharev and A. Nitzan, Optics of exciton-plasmon nanomaterials, J. Phys.: Condens. Matter 29, 443003 (2017).
[4] M. Ramezani, M. Berghuis, and J. G. Rivas, Strong lightmatter coupling and exciton-polariton condensation in lattices of plasmonic nanoparticles, J. Opt. Soc. Am. B 36, E88 (2019).

[5] A. F. Kockum, A. Miranowicz, S. De Liberato, S. Savasta, and F. Nori, Ultrastrong coupling between light and matter, Nat. Rev. Phys. 1, 19 (2019).

[6] V. N. Pustovit and T. V. Shahbazyan, Plasmon-mediated superradiance near metal nanostructures, Phys. Rev. B 82, 075429 (2010). 
[7] V. V. Temnov and U. Woggon, Superradiance and Subradiance in an Inhomogeneously Broadened Ensemble of Two-Level Systems Coupled to a Low- $Q$ Cavity, Phys. Rev. Lett. 95, 243602 (2005).

[8] M. I. Stockman, The spaser as a nanoscale quantum generator and ultrafast amplifier, J. Opt. 12, 024004 (2010).

[9] P. Berini and I. De Leon, Surface plasmon-polariton amplifiers and lasers, Nat. Photon. 6, 16 (2012).

[10] W. Zhou, M. Dridi, J. Y. Suh, C. H. Kim, D. T. Co, M. R. Wasielewski, G. C. Schatz, T. W. Odom et al., Lasing action in strongly coupled plasmonic nanocavity arrays, Nat. Nanotechnol. 8, 506 (2013).

[11] M. Richter, M. Gegg, T. S. Theuerholz, and A. Knorr, Numerically exact solution of the many emitter-cavity laser problem: Application to the fully quantized spaser emission, Phys. Rev. B 91, 035306 (2015).

[12] Y. Zhang and V. May, Theory of molecule metal nano-particle interaction: Quantum description of plasmonic lasing, J. Chem. Phys. 142, 224702 (2015).

[13] J.-P. Martikainen, M. O. J. Heikkinen, and P. Törmä, Condensation phenomena in plasmonics, Phys. Rev. A 90, 053604 (2014).

[14] S. Zaster, E. R. Bittner, and A. Piryatinski, Quantum symmetry breaking of exciton/polaritons in a metal-nanorod plasmonic array, J. Phys. Chem. A 120, 3109 (2016).

[15] T. K. Hakala, A. J. Moilanen, A. I. Väkeväinen, R. Guo, J.-P. Martikainen, K. S. Daskalakis, H. T. Rekola, A. Julku, and P. Törmä, Bose-Einstein condensation in a plasmonic lattice, Nat. Phys. 14, 739 (2018).

[16] R. H. Lehmberg, Radiation from an $N$-atom system. I. General formalism, Phys. Rev. A 2, 883 (1970).

[17] F. C. Spano and S. Mukamel, Superradiance in molecular aggregates, J. Chem. Phys. 91, 683 (1989).

[18] N. Shammah, S. Ahmed, N. Lambert, S. De Liberato, and F. Nori, Open quantum systems with local and collective incoherent processes: Efficient numerical simulations using permutational invariance, Phys. Rev. A 98, 063815 (2018).

[19] M. B. Plenio and P. L. Knight, The quantum-jump approach to dissipative dynamics in quantum optics, Rev. Mod. Phys. 70, 101 (1998).

[20] A. Metelmann and A. A. Clerk, Nonreciprocal Photon Transmission and Amplification via Reservoir Engineering, Phys. Rev. X 5, 021025 (2015).

[21] F. Verstraete, M. M. Wolf, and J. I. Cirac, Quantum computation and quantum-state engineering driven by dissipation, Nat. Phys. 5, 633 (2009).

[22] M. J. Kastoryano, F. Reiter, and A. S. Sørensen, Dissipative Preparation of Entanglement in Optical Cavities, Phys. Rev. Lett. 106, 090502 (2011).

[23] F. Reiter, D. Reeb, and A. S. Sørensen, Scalable Dissipative Preparation of Many-Body Entanglement, Phys. Rev. Lett. 117, 040501 (2016).
[24] H. Li, A. Piryatinski, A. R. Srimath Kandada, C. Silva, and E. R. Bittner, Photon entanglement entropy as a probe of manybody correlations and fluctuations, J. Chem. Phys. 150, 184106 (2019).

[25] S. Diehl, A. Micheli, A. Kantian, B. Kraus, H. Büchler, and P. Zoller, Quantum states and phases in driven open quantum systems with cold atoms, Nat. Phys. 4, 878 (2008).

[26] Y. Hama, W. J. Munro, and K. Nemoto, Relaxation to Negative Temperatures in Double Domain Systems, Phys. Rev. Lett. 120, 060403 (2018).

[27] P. Kirton, M. M. Roses, J. Keeling, and E. G. Dalla Torre, Introduction to the Dicke model: From equilibrium to nonequilibrium, and vice versa, Adv. Quantum Technol. 2, 1800043 (2019).

[28] P. Kirton and J. Keeling, Superradiant and lasing states in driven-dissipative Dicke models, New J. Phys. 20, 015009 (2018).

[29] Y. Shchadilova, M. M. Roses, E. G. Dalla Torre, M. D. Lukin, and E. Demler, Fermionic formalism for driven-dissipative multilevel systems, Phys. Rev. A 101, 013817 (2020).

[30] C. W. Gardiner and P. Zoller, Quantum Noise (Springer, New York, 2004).

[31] S. Mukamel, Principles of Nonlinear Optical Spectroscopy (Oxford University Press, Oxford, 1995).

[32] H. Li, A. Piryatinski, J. Jerke, A. R. S. Kandada, C. Silva, and E. R. Bittner, Probing dynamical symmetry breaking using quantum-entangled photons, Quantum Sci. Technol. 3, 015003 (2018).

[33] W. Kopylov, C. Emary, and T. Brandes, Counting statistics of the Dicke superradiance phase transition, Phys. Rev. A 87, 043840 (2013).

[34] Z. Zhiqiang, C. H. Lee, R. Kumar, K. Arnold, S. J. Masson, A. Parkins, and M. Barrett, Nonequilibrium phase transition in a spin-1 Dicke model, Optica 4, 424 (2017).

[35] F. Dimer, B. Estienne, A. S. Parkins, and H. J. Carmichael, Proposed realization of the Dicke-model quantum phase transition in an optical cavity QED system, Phys. Rev. A 75, 013804 (2007).

[36] C. Cohen-Tannoudji, J. Dupont-Roc, and G. Grynberg, Photons and Atoms (Wiley-VCH, Weinheim, 2014).

[37] P. W. Milonni, The Quantum Vacuum (Academic, New York, 1994).

[38] C. W. Gardiner and M. J. Collett, Input and output in damped quantum systems: Quantum stochastic differential equations and the master equation, Phys. Rev. A 31, 3761 (1985).

[39] P. W. Milonni, D. F. V. James, and H. Fearn, Photodetection and causality in quantum optics, Phys. Rev. A 52, 1525 (1995).

[40] P. W. Milonni, An Introduction to Quantum Optics and Quantum Fluctuations (Oxford University Press, New York, 2019). 